\title{
Numerical Analysis of Surface Chemistry in High-Enthalpy Flows
}

\author{
Abhilasha Anna* and Iain D. Boyd $\ddagger$ \\ University of Michigan, Ann Arbor, Michigan 48109
}

DOI: $10.2514 / 1 . T 4530$

\begin{abstract}
The effects of surface-chemistry processes of a graphite sample exposed to a subsonic high-enthalpy nitrogen flow are investigated using a coupled computational fluid-dynamics/surface-chemistry model. The results obtained are assessed for the accuracy of the model using experimental data from tests conducted in a $30 \mathrm{~kW}$ inductively coupled plasma torch facility at the University of Vermont. Significant discrepancies are observed between the computational and experimental results. Therefore, a study is performed to determine sensitivities of flow and surface parameters to variations in testing input conditions, as well as physical modeling parameters. Measurements of the absolute number density are required to draw firm conclusions about the surface-chemistry models, as well as the surface reactions involved.
\end{abstract}

\section{Nomenclature}

$C_{k}=$ concentration of gas species $k, \mathrm{~mol} / \mathrm{m}^{3}$

$D_{k} \quad=$ diffusion coefficient of species $k, \mathrm{~m}^{2} / \mathrm{s}$

$E_{\text {ad }} \quad=$ energy barrier for adsorption, $\mathrm{J} / \mathrm{mol}$

$E_{\mathrm{ER}} \quad=$ energy barrier for Eley-Rideal recombination, $\mathrm{J} / \mathrm{mol}$

$h \quad=$ species enthalpy, $\mathrm{J} / \mathrm{kg}$

$K=$ total number of species

$K_{g} \quad=$ total numbers of gas species

$K_{n b} \quad=$ number of species in bulk phase $n b$

$K_{n s, n a}=$ number of species in active site set $n a$ on surface phase $n s$

$k_{B} \quad=$ Boltzmann constant

$k_{f i}, k_{b i}=$ forward and backward reaction rates for reaction $i$

$M_{k} \quad=$ molar weight of species $k, \mathrm{~kg} / \mathrm{mol}$

$\dot{m}_{b} \quad=$ mass blowing rate due to surface reactions, $\mathrm{kg} / \mathrm{m}^{2} / \mathrm{s}$

$N=$ total number of phases

$N_{n b} \quad=\quad$ number of bulk species

$N_{g}, N_{s}, N_{b}=$ number of gas, surface, and bulk phases

$N_{n s, a}=$ number of active site sets in surface phase $n s$

$N_{R} \quad=$ number of surface reactions

$P \quad=$ pressure, $\mathrm{Pa}$

$q \quad=$ total heat flux, $\mathrm{W} / \mathrm{m}^{2}$

$q_{\text {conv }}=$ convective heat flux, $\mathrm{W} / \mathrm{m}^{2}$

$q_{\text {diff }}=$ diffusive heat flux, $\mathrm{W} / \mathrm{m}^{2}$

$R_{u} \quad=$ universal gas constant, $\mathrm{J} / \mathrm{mol} / \mathrm{K}$

$r_{i, n s}$

$S, S_{0}$

$T$

$\nu_{k i}$

$\nu_{s}$

$\bar{\nu}_{k}$

$\nu_{k i}^{\prime}$

$=$ reaction flux of reaction $i$ on surface phase $n s$, $\mathrm{mol} / \mathrm{m}^{2} / \mathrm{s}$

$=$ sticking coefficient

$=$ translational-rotational temperature, $\mathrm{K}$

$=$ net stoichiometric coefficient for species $k$ in reaction $i$

$=$ sum of the stoichiometric coefficients of all surface reactants

$=$ thermal speed of gas-phase species $k, \mathrm{~m} / \mathrm{s}$

$=$ reactant stoichiometric coefficient for species $k$ in reaction $i$

Received 9 July 2014; revision received 4 March 2015; accepted for publication 6 March 2015; published online 24 April 2015. Copyright @ 2015 by the American Institute of Aeronautics and Astronautics, Inc. All rights reserved. Copies of this paper may be made for personal or internal use, on condition that the copier pay the $\$ 10.00$ per-copy fee to the Copyright Clearance Center, Inc., 222 Rosewood Drive, Danvers, MA 01923; include the code 1533-6808/15 and \$10.00 in correspondence with the CCC.

*Postdoctoral Research Fellow, Department of Aerospace Engineering. Member AIAA.

$\dagger$ James E. Knott Professor, Department of Aerospace Engineering. Fellow AIAA.

\begin{tabular}{|c|c|c|}
\hline$\nu_{k i}^{\prime \prime}$ & $=$ & $\begin{array}{l}\text { product stoichiometric coefficient for species } k \text { in } \\
\text { reaction } i\end{array}$ \\
\hline$\dot{w}_{k}$ & $=$ & $\begin{array}{l}\text { production rate of species } k \text { in all reactions, } \\
\mathrm{mol} / \mathrm{m}^{2} / \mathrm{s}\end{array}$ \\
\hline$\dot{w}_{k i}$ & $=$ & $\begin{array}{l}\text { production rate of species } k \text { in reaction } i \text {, } \\
\mathrm{mol} / \mathrm{m}^{2} / \mathrm{s} \text {, }\end{array}$ \\
\hline$Y$ & $=$ & mass fraction \\
\hline$\Gamma_{k}$ & $=$ & impingement flux of gas species $k, \mathrm{~m}^{2} / \mathrm{s}$ \\
\hline$\gamma$ & $=$ & reaction efficiency \\
\hline$\theta_{n s, k}$ & $=$ & $\begin{array}{l}\text { fraction of active sites occupied by species } k \text { on } \\
\text { surface phase } n s\end{array}$ \\
\hline$\rho$ & $=$ & density, $\mathrm{kg} / \mathrm{m}^{3}$ \\
\hline$\sigma$ & $=$ & Stefan-Boltzmann constant, $\mathrm{W} / \mathrm{m}^{2} / \mathrm{K}^{-4}$ \\
\hline$\Phi_{n s}$ & $=$ & active site density on surface phase $n s, \mathrm{~mol} / \mathrm{m}^{2}$ \\
\hline$\Phi_{n s, k}$ & $=$ & $\begin{array}{l}\text { concentration of species } k \text { on surface phase } n s \text {, } \\
\mathrm{mol} / \mathrm{m}^{2}\end{array}$ \\
\hline$\chi_{k}$ & $=$ & mole fraction of species $k$ \\
\hline 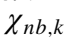 & $=$ & mole fraction of bulk species $k$ in bulk phase \\
\hline
\end{tabular}

Subscripts

$b \quad=$ bulk phase

$e \quad=$ empty site

$g \quad=$ gas phase

na $\quad=$ number of active sites

$n b \quad=$ number of bulk phases

$n s \quad=$ number of surface phases

$s \quad=\quad$ surface phase

$\operatorname{tr}=$ translational-rotational energy mode

ve $\quad=$ vibrational-electronic energy mode

$w \quad=$ wall value

$\infty \quad=$ reference freestream conditions

\section{Introduction}

$\mathbf{H}$ YPERSONIC vehicles experience heating during high-speed flight through the atmosphere that cause very high temperatures on their surface. When a vehicle or probe enters the atmosphere of any planet at hypersonic speeds, a bow shock is formed in its front. The kinetic energy of the hypersonic flow is converted into internal energy of the gas that creates very high temperatures in the shock layer [1]. These temperatures are high enough to excite the vibrational energy mode within the molecules, as well as cause dissociation chemistry. Therefore, such vehicles use a thermal protection system (TPS) that provides insulation from the severe aerodynamic heating encountered during hypersonic flight through a planetary atmosphere. The TPS is a single point-of-failure system, as the prolonged exposure to high temperature can cause the materials to fail $[2,3]$. The need to design a reliable TPS necessitates good understanding of the physical and chemical processes that determine the aerothermal heating environment. 
Depending on the heating encountered during hypersonic flight, an ablative or nonablative TPS may be used [3]. Nonablative or reusable materials (e.g., ceramic tiles used on the space shuttle with a peak heating [4] of $60 \mathrm{~W} / \mathrm{cm}^{2}$ ) are used where the reentry conditions are relatively mild. In addition to the entry velocity, the heating is also dependent on the entry flight-path angle that is determined by the trajectory of the vehicle [5]. An ablative TPS is used where relatively high heating rates are generated during reentry (e.g., the heat shield for the Stardust mission with a peak heating [6] of $942 \mathrm{~W} / \mathrm{cm}^{2}$ ). Ablative TPS materials accommodate high heating rates and heat loads through phase change and mass loss. The ablative material absorbs the heat and leaves the hypersonic vehicle as the material is consumed and ablates away. It is designed to slowly recess in a controlled manner. An ablative TPS has been used for most planetary entry probes and high-velocity Earth atmosphere reentry vehicles, including Stardust, the Mars Science Laboratory, Apollo, etc. Ablative TPS materials are usually of two types, viz., pyrolyzing and nonpyrolyzing ablators. Pyrolyzing ablators (also referred to as charring ablators) are reinforced composites that have polymer resins as binders. An example of a charring TPS material is phenolicimpregnated carbon ablator.

Nonpyrolyzing ablators (also referred to as noncharring ablators) are those that withstand heat by losing mass only by surface ablation and mechanical erosion [7,8]. Examples of noncharring TPS materials are carbon-carbon and silica. Very high temperatures in the shock layer may cause the molecular species to dissociate. The TPS material can act as a catalyst and, if dissociated atoms diffuse to the surface, recombination of dissociated boundary-layer species may occur, which increases the convective heating to the surface. Thus, a less catalytic surface is desirable to minimize this additional heating. Also, when the vehicle surface is heated, the surface material may chemically react with the boundary-layer gases, leading to surface recession as a result of surface material consumption. These chemical reactions can be endothermic (vaporization, sublimation) or exothermic (oxidation, nitration) and will affect the net heating to the surface. Catalycity of an ablative TPS material and surface-participating reactions that lead to surface recession are key factors that impact the heating of the vehicle surface. Therefore, detailed studies of these interactions that occur between the surface and the atmosphere gas are required for the accurate prediction of aerothermal heating of the vehicle TPS and in characterizing TPS materials.

The major objective of this work is to investigate surfacechemistry processes using a coupled computational fluid-dynamics/ surface-chemistry model and assess the accuracy of the model using experimental data. Significant discrepancies are observed between the computational and experimental results. Therefore, another objective is to understand the sensitivities of flow and surface parameters to variations in testing input conditions. A sensitivity study is performed to understand the effects of various inputs, as well as physical modeling parameters on the subsonic high-enthalpy nitrogen flow in the boundary layer in front of a graphite sample and its surface properties.

The layout of this paper is as follows. The paper comprises six sections, with the Introduction being Sec. I. Section II outlines the technical approach used in this work. It describes the experimental and computational techniques that are used to study the gas-surface interactions that occur on a vehicle surface during its entry into a planetary atmosphere. Section III presents a description of the numerical boundary conditions used in the code. Section IV presents a description of the numerical setup used in this study.

Section $\mathrm{V}$ presents the results obtained from the numerical simulations of the experimental configuration performed using the computational fluid dynamics (CFD) code LeMANS and their comparison with the experimental data. The results obtained from the analysis performed for sensitivity of boundary-layer flow parameters and surface properties to different chemical compositions at the inlet of the inductively coupled plasma (ICP) torch exit are also presented, in addition to the effects of including the conduction within the sample wall in the calculations. The paper ends with conclusions in Sec. VI.

\section{Technical Approach}

Computational fluid dynamic models can be used for simulating environments that cannot be studied in an experimental test facility. These models can be used for accurately predicting the aerothermal environment of the vehicle TPS during entry, but these models can be used to perform such analysis only after they have been validated for physical accuracy by comparison with experimental measurements. Both computational and experimental methods can be used collectively to understand the physical and chemical processes that determine the aerodynamic heating of a probe or hypersonic vehicle during its entry into a planetary atmosphere.

This section describes the experimental and computational techniques that are used to study the gas-surface interactions that occur on a vehicle surface during its entry into a planetary atmosphere. The section first provides a brief description of the experimental facility at the University of Vermont, followed by an overview of the CFD code used in this work along, with the description of gas-surface interaction models implemented in the code.

\section{A. Experimental Facility}

Experimental tests were conducted by Professor Doug Fletcher and his graduate students in a $30 \mathrm{~kW}$ inductively coupled plasma torch facility at the University of Vermont $[9,10]$. Laser diagnostic instrumentation that employs a laser-induced fluorescence (LIF) technique is installed at the facility. LIF is capable of measuring various flow parameters such as translational temperature and species number densities at different locations in the flowfield. This section describes the facility and techniques that are used to obtain experimental data that will be compared to the numerical results.

The ICP torch facility is designed to test scaled material samples in high-enthalpy gas flows for simulation of planetary entry and Earth atmosphere reentry trajectory heating conditions. It is configured for operation with subsonic flow to simulate postshock conditions of high-enthalpy flight for a stagnation point geometry. The facility test conditions can be extrapolated to flight conditions by matching three parameters at the edge of the boundary layer, i.e., the flight total enthalpy, the stagnation pressure, and the velocity gradient. The methodology is known as local heat transfer simulation and is performed under the assumption of local thermochemical equilibrium. It is discussed in detail in [11-13]. The stagnation point heat flux in the flight is equal to that in ground tests if these parameters are matched [14].

The facility primarily comprises the power supply unit, gas injection system, and plasma test chamber. The gas-injection system provides the nitrogen gas at room temperature that enters into the quartz confinement tube where hot nitrogen plasma is generated through an induced RF magnetic field created by a helical load coil.

The hot nitrogen plasma then flows out of the quartz tube from the top into the test chamber of the ICP facility where the sample is tested. The test chamber is constructed from stainless steel, and the torch locations with the highest heat loads are actively cooled with a closed-loop water system. The test sample is installed in a brass sample holder, and the back space side of the sample is water cooled. For this investigation, experimental results from graphite samples tested in the nitrogen plasma stream are used. The test samples are constructed from DFP2-grade graphite, fabricated by Poco Graphite, Inc. [15]. Most ablative heat shields are designed from carbon-based matrix materials impregnated with low-temperature phase change polymer resins that pyrolyze, leaving a carbon-rich char layer. Since the carbon layer continues to interact with the boundary-layer gases, reactions between this layer and the gas-phase particles are of immense interest, and therefore graphite is used for this study. Graphite is noncharring, and therefore pyrolysis gases are not produced. Figure 1 shows a photograph of the graphite sample during exposure to the nitrogen plasma in the test chamber of the ICP torch facility. The test sample is a 25 -mm-diam graphite sample mounted at a distance of $90 \mathrm{~mm}$ from the quartz tube exit.

The quantities measured are the surface heat flux, surface temperature, relative nitrogen atom number density, and translational temperature in the reacting boundary layer above the graphite surface. 


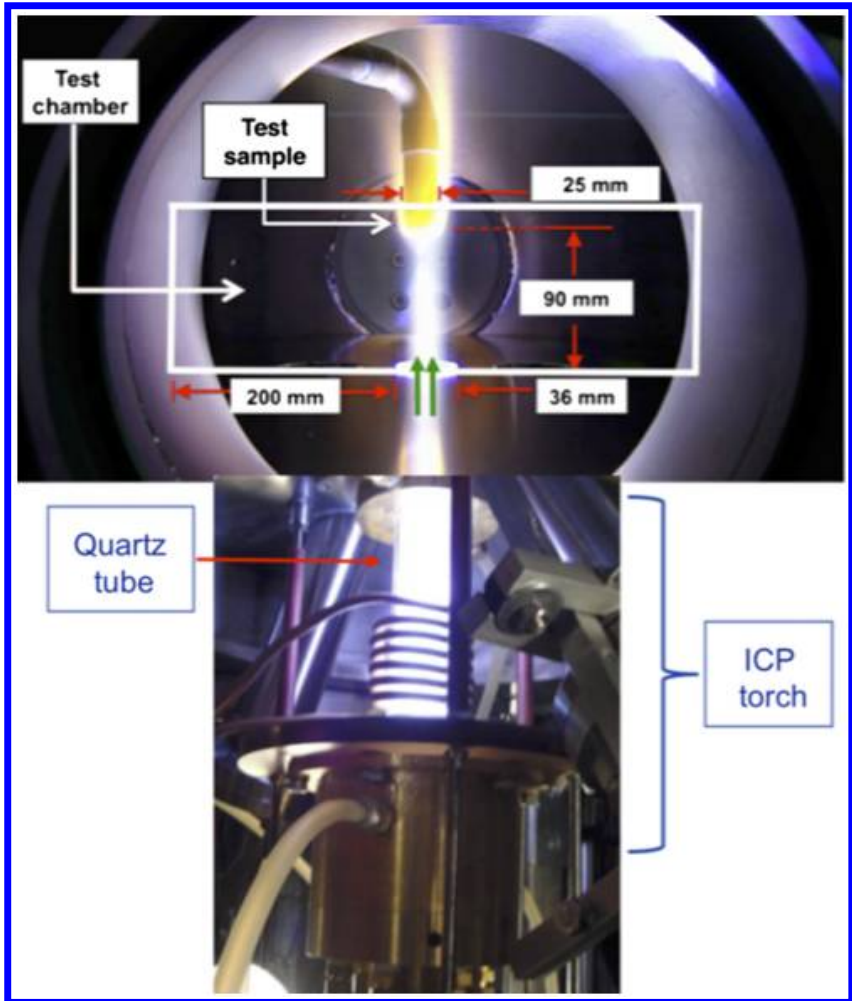

Fig. 1 Experimental setup (section in box is simulated using the CFD code LeMANS).

The stagnation region heat transfer is measured with a copper slug calorimeter [16]. The copper slug is identical in size and shape to the graphite test sample. It is exposed to identical freestream conditions as the graphite sample when the measurements are made. The test sample surface temperature is measured using a two-color infrared optical pyrometer with a temperature range from 1273 to $3273 \mathrm{~K}$. The experimental tests measure the gas-phase flow properties (i.e., nitrogen atom number density and translational temperature) in the reacting boundary layer above the graphite surface using a twophoton laser-induced fluorescence technique. Unfortunately, the calibrated absolute atom number density values are not yet available. Therefore, the relative nitrogen atom number density is used for assessing the computational simulations. The relative nitrogen atom number density is the ratio of the spectrally integrated LIF signal for the ICP test chamber and the microwave discharge flow reactor (MDFR). A MDFR is used to establish absolute species concentration and translational temperature in the flow in the ICP test chamber. A detailed explanation of the technique for the quantities measured is provided in $[9,17,18]$. The graphite sample mass loss (ablation) rate is also quantified from pre- and posttest mass measurements.

\section{B. Numerical Method}

The numerical simulations in this work are conducted using the Navier-Stokes computational fluid dynamics code LeMANS [19], developed at the University of Michigan. It is a general-purpose parallel three-dimensional code that solves the laminar NavierStokes equations, including chemical and thermal nonequilibrium effects on unstructured computational grids. The flow is modeled assuming the continuum approximation is valid. The translational and rotational energy modes of all species can be described by their respective temperatures, $T$ and $T_{r}$, in the code. However, in this work, the translational and rotational modes are assumed to be equilibrated, as these modes usually require relatively few collisions to reach equilibrium. Therefore, these modes for all species are described by a single temperature $T_{\mathrm{tr}}$. The vibrational and electronic energy modes

\footnotetext{
TPersonal conversation with A. J. Lutz, University of Vermont, Mechanical Engineering, Burlington, VT, March 2013.
}

of all species are described by a single temperature $T_{\text {ve }}$ [20]. The production and consumption rates of species are modeled using a finite rate chemistry model developed by Martin and Boyd [21], along with Park's two-temperature model [22], to account for thermal nonequilibrium effects on the reaction rates.

The set of partial differential equations is solved using a finite volume method on unstructured grids. The inviscid fluxes across cell faces are discretized using a modified form of the Steger-Warming flux vector splitting scheme [23], which is less dissipative and produces better results in boundary layers than their original scheme. The viscous terms are calculated using a centered scheme. Time integration is performed using a point implicit or a line implicit method. LeMANS is parallelized using METIS [24], which partitions the computational grid between the processors and the message passage interface protocol to communicate information between processors. Turbulent flows could be simulated for simple geometries using a zero equation algebraic Baldwin-Lomax turbulence model [25] implemented in LeMANS. The model is not used for numerical simulation in this work, as the flowfield studied has laminar behavior. The freestream Reynolds number for the flowfield analyzed in this work is 295 , indicating that the flow is laminar. The mixture transport properties (i.e., the coefficients of viscosity, thermal conductivity, and mass diffusion) can be computed using two models. The first model uses Wilke's semiempirical mixing rule [26], with species viscosities calculated using Blottner et al.'s model [27]; and the species thermal conductivities are determined using Eucken's relation [28]. The other model uses Gupta et al.'s mixing rule [29], with species viscosities and thermal conductivities calculated using collision cross-section data. Details on the modeling of these equations can be found in [30]. LeMANS can simulate twodimensional/axisymmetric flows using any mixture of quadrilaterals and triangles, as well as three-dimensional flows using any mixture of hexahedrals, tetrahedrals, prisms, and pyramids. The code has been extensively validated for hypersonic flows [30-33].

\section{Numerical Boundary Conditions}

\section{A. Inlet and Outlet Boundary Conditions}

In LeMANS, befoer this work, the inflow and outflow boundary conditions were specified only for hypersonic flows. For hypersonic flows, all the variables should be specified at the inflow and none should be specified at the outflow when the exit is also at supersonic speeds. The flow in the case of an ICP torch test facility is subsonic in nature. For a subsonic flow, the disturbances propagate upstream against the flow direction, and this needs to be accounted for. Therefore, new inflow and outflow boundary conditions are implemented for subsonic flow conditions [34]. For the subsonic inlet boundary, the freestream state is fully specified. In this condition, all the variables (i.e., velocity, density, and temperature) are specified as input.

For the subsonic outlet boundary condition, a constant pressure boundary condition is implemented in which static pressure is specified at the outlet. The velocity and density variables are solved using zeroth-order extrapolation. The specified outlet pressure is used to compute the temperature variable using the equation of state.

\section{B. Wall Boundary Conditions}

In the simplest approach, wall catalycity effects are accounted for in LeMANS by choosing a noncatalytic or a supercatalytic surface as the species boundary condition. The catalycity of a surface in general can be accounted for by four types of conditions at the wall boundary, as described in Table $\underline{1}$. A supercatalytic boundary condition is

Table 1 Species boundary conditions

\begin{tabular}{lc}
\hline \hline Boundary condition & Description \\
\hline $\begin{array}{l}\text { Noncatalytic } \\
\text { Supercatalytic }\end{array}$ & $\begin{array}{c}\text { No recombination of atoms on the surface } \\
\text { Atoms that strike the surface recombine } \\
\text { to the freestream gas composition }\end{array}$ \\
$\begin{array}{l}\text { Fully catalytic } \\
\text { All atoms that strike the surface recombine } \\
\text { to form molecules }\end{array}$ \\
\hline \hline
\end{tabular}


inappropriate for the flows that comprise dissociated species at the freestream, as this condition will lead to the same composition through dissociation/recombination at the wall as in the freestream, which is undesirable for conditions where the effect of recombination on the heat transfer is to be studied. In addition to surface catalysis, surface participating reactions are required to be included in the analysis for a thorough understanding of gas-surface interactions. Therefore, a simple binary catalytic recombination model and a complex finite rate surface-chemistry model are implemented in LeMANS.

\section{Binary Catalytic Recombination Model}

For the simulation of the full range of catalycity regimes, from a noncatalytic wall to a fully catalytic wall, a simplified catalytic atom recombination model (i.e., a binary interaction model with full energy accommodation [35]) is implemented in LeMANS. It is a simple model applied to a binary gaseous mixture of atoms and molecules of the same species. It is implemented by balancing the mass flux of the relevant species at the wall. It is applied as a species boundary condition by considering a first-order recombination reaction for a binary gas at the wall. It only accounts for catalytic homogeneous recombination at the surface.

\section{Finite Rate Surface-Chemistry Model}

The finite rate surface-chemistry (FRSC) model is a general gassurface interaction model [36-38]. It can be used to investigate the effects of surface catalysis as well as surface participating reactions. The FRSC model developed by Marschall and MacLean [36] and MacLean et al. [37] was implemented in LeMANS by Alkandry et al. [38]. The model can simulate the chemical reactions between the hypersonic gas and surface of the vehicle during planetary entry. A simplified binary catalytic atom recombination model can only be used to study the effects of surface catalysis for a constant catalytic efficiency applied to a binary gaseous mixture of atoms and molecules. The FRSC model can be applied to multiple gaseous species and can account for different surface reactions, such as particle adsorption/desorption, the recombination of an atom of the gas with an atom adsorbed on the wall [Eley-Rideal (E-R) reaction], recombination of two adsorbed atoms at the wall (Langmuir-Hinshelwood reaction), and reactions leading to surface recession (e.g., carbon nitridation and oxidation). The FRSC model is based on the concept of simulating surface chemical reactions by competing finite rate processes. It accounts for catalytic heterogeneous recombination at the surface.

It comprises three environments, viz., gas, surface, and bulk (solid) environments. Each environment can consist of one or more "phases" that correspond to a distinct physical region of the respective environment. The gas environment is a single phase $\left(N_{g}=1\right)$ that contains gas-phase species.

The surface environment can consist of multiple phases represented by $n s$ ranging from one to the total number of surface phases $N_{s}$. Each surface phase occupies a fraction $\Omega_{n s}$ of the total surface. Each surface phase can comprise multiple sets of active sites represented by $n a$ ranging from one to the total number of active sites for each phase $N_{n s, a}$. Each active site set has a site density $\Phi_{n s, n a}$. All the surface reactions take place at active sites. Similarly, the bulk environment can consist of multiple phases $\left(n b=1, \ldots, N_{b}\right)$, where $n b$ is the number of bulk phases. Each phase occupies a volume fraction $v_{n b}$ of the bulk and contains a unique set of species $K_{n b}$. The total number of phases $N$ is

$$
N=1+N_{s}+N_{b}
$$

The total number of species is the summation of gas, surface, and bulk phase species given by Eq. (2). In this formulation, a particular species is considered a different species if it is in gas phase or in a particular active site in a surface phase or in a bulk phase. The description of all the variables is provided in the nomenclature:

$$
K=K_{g}+\sum_{n s=1}^{N_{s}} \sum_{n a=1}^{N_{n s, a}} K_{n s, n a}+\sum_{n b=1}^{N_{b}} K_{n b}
$$

For a system with $K$ species and $N_{R}$ surface reactions, the general form of the surface reaction $i$ can be expressed as

$$
\sum_{k=1}^{K} \nu_{k i}^{\prime} A_{k} \rightleftharpoons \sum_{k=1}^{K} \nu_{k i}^{\prime \prime} A_{k}
$$

where $\nu_{k i}^{\prime}$ and $\nu_{k i}^{\prime \prime}$ are the respective reactant and product stoichiometric coefficients for species $A_{k}$. The net production rate $\dot{w}_{k}$ of species $A_{k}$ is the sum of the production rates from all surface reactions given by Eq. (4). It applies to species in any phase at the gas/surface interface:

$$
\dot{w}_{k}=\sum_{i=1}^{N_{R}} \dot{w}_{k i}
$$

where the reaction-specific production rate $\dot{w}_{k i}$ is the product of net stoichiometric coefficient $\nu_{k i}$ and reaction flux $r_{i, n s}$ for reaction $i$ on phase $n s$ given by the expression in Eq. (5):

$$
\begin{aligned}
\dot{w}_{k i} & =\nu_{k i} r_{i, n s} \\
\nu_{k i} & =\left(\nu_{k i}^{\prime \prime}-\nu_{k i}^{\prime}\right) \\
r_{i, n s} & =k_{f i} \prod_{k=1}^{K} X_{k}^{\nu_{k i}^{\prime}}-k_{b i} \prod_{k=1}^{K} X_{k}^{\nu_{k i}^{\prime \prime}}
\end{aligned}
$$

where $k_{f i}$ and $k_{b i}$ are the forward and backward reaction rates for reaction $i$, respectively. $X_{k}$ is the concentration of species $A_{k}$ at the surface and, for each phase, it can be described as

Gas phase:

$$
X_{k}=C_{k}=\chi_{k} \frac{P}{R T}
$$

Surface phase:

$$
X_{k}=\Phi_{n s, k}=\theta_{n s, k} \Phi_{n s}
$$

Bulk phase:

$$
X_{k}=\chi_{n b, k}
$$

The forward reaction rate for each surface reaction type can be specified by an Arrhenius function or using a kinetic-based formulation for specific processes like adsorption, Eley-Rideal recombination, and Langmuir-Hinshelwood recombination. The FRSC model can account for competing finite rate processes under a given set of experimental conditions and provides an effective reaction efficiency for a gas-phase reactant consumed in a surface reaction process. For this study, the FRSC model is used to simulate a constant reaction efficiency by using the appropriate choice of reaction types and parameters. The gas-surface interaction processes studied are the recombination of nitrogen atoms to molecules at the surface due to catalysis and the carbon nitridation reaction where nitrogen atoms react with the surface carbon to form gaseous $\mathrm{CN}$. The surface reaction types considered are adsorption and Eley-Rideal recombination to emulate a constant reaction efficiency for these processes. The E-R mechanism involves the reaction of a gas-phase species with an adsorbed species to form a gas-phase product. The surface reaction for an adsorption process for a particle $A$ can be represented by

$$
A+(s) \rightarrow A(s)
$$

where $(s)$ is an empty active site, and $A(s)$ is an adsorbed particle. The forward reaction flux for an adsorption process is the product of the 
sticking coefficient $S_{0}$, the impingement flux $\Gamma_{A}$ of species $A$ on the surface, and the fraction $\theta_{s, e}$ of available active sites that are empty:

$$
r_{f}=S \Gamma_{A} \theta_{s, e}
$$

where the sticking coefficient is

$$
S=S_{0} \exp \left(\frac{-E_{\mathrm{ad}}}{R T}\right)
$$

The sticking or adsorption coefficient $S_{0}$ is the fraction of the gasphase species that hits the surface and becomes adsorbed.

The impingement flux is given by

$$
\Gamma_{A}=\frac{\bar{\nu}_{A}}{4} C_{A}
$$

where the thermal velocity of species $A$ is

$$
\bar{\nu}_{A}=\sqrt{\frac{8 R u T}{\pi M_{A}}}
$$

and the fraction of available empty active sites is

$$
\theta_{s, e}=\frac{\Phi_{s, e}}{\Phi_{s}}
$$

The forward reaction rate for an adsorption process is expressed by the following:

$$
k_{f}=\left[\frac{\bar{\nu}_{A}}{4 \Phi_{s}^{\nu_{s}}}\right] S_{0} \exp \left(-\frac{E_{\mathrm{ad}}}{R_{u} T}\right)
$$

The surface reaction for an Eley-Rideal recombination of a particle $A$ with an adsorbed particle $B(s)$ can be represented by

$$
A+B(s) \rightarrow A B+(s)
$$

The forward reaction flux for an Eley-Rideal recombination process is the product of the Eley-Rideal reaction efficiency $\gamma_{\mathrm{ER}}$, the impingement flux $\Gamma_{A}$ of species $A$ on the surface, and the fraction $\theta_{s, B}$ of available active sites that are occupied by adsorbed species $B$ :

$$
r_{f}=\gamma_{\mathrm{ER}} \Gamma_{A} \theta_{s, B}
$$

where the Eley-Rideal reaction efficiency is

$$
\gamma_{\mathrm{ER}}=\gamma_{0} \exp \left(\frac{-E_{\mathrm{ER}}}{R T}\right)
$$

and the fraction of available active sites is

$$
\theta_{s, B}=\frac{\Phi_{s, B}}{\Phi_{s}}
$$

The forward reaction rate for an Eley-Rideal recombination process is expressed by the following:

$$
k_{f}=\left[\frac{\bar{\nu}_{A}}{4 \Phi_{s}^{\nu_{s}}}\right] \gamma_{0} \exp \left(-\frac{E_{\mathrm{ER}}}{R_{u} T}\right)
$$

The backward reaction rate for both processes is zero, as both the thermal desorption and dissociation with a partial adsorption process [shown by Eq. (12)] are not considered in this work:

Thermal desorption:

$$
A(s) \rightarrow A+(s)
$$

Dissociation with partial adsorption:

$$
A B+(s) \rightarrow A+B(s)
$$

The E-R reaction can also be used to represent a process where a gas-phase species impinges on the surface and reacts with the surface. An example is shown in Eq. (13), where the gas-phase species $A$ impinges on the surface and reacts with the bulk phase species $B_{b}$ on the surface:

$$
A+(s)+B_{b} \rightarrow A B+(s)
$$

This equation is used to emulate the carbon nitridation reaction.

The species mass fraction at the wall is calculated by balancing the mass flux of the relevant species, taking the consumption and production at the wall into account as shown in Eq. (14):

$$
-\left.\rho_{w} D_{k} \frac{\partial Y_{k}}{\partial n}\right|_{w}+\rho_{w} v_{w} Y_{k, w}=M_{k} \dot{w}_{k}
$$

Here, the first term

$$
-\left.\rho_{w} D_{k} \frac{\partial Y_{k}}{\partial n}\right|_{w}
$$

represents diffusion of the gas-phase species, the second term $\rho_{w} v_{w} Y_{k, w}$ represents the mass flux of species blown from the surface into gas phase, and the term on the right $M_{k} \dot{w}_{k}$ represents production or consumption of species from surface reactions. In the second term, $\rho_{w} v_{w}$ is the mass blowing rate $\dot{m}_{b}$ at the surface due to surface reactions (e.g., oxidation, nitridation, and sublimation). The velocity of the blowing mass is $v_{w}$. It is given by the following expression:

$$
\dot{m}_{b}=\rho_{w} v_{w}=-\sum_{n b=1}^{N_{n b}} \sum_{k=1}^{K_{n b}} M_{k} \dot{w}_{k}
$$

\section{Heat Flux at the Wall}

Heat transfer to the surface is composed of convective heat flux and heat flux due to diffusion of species to the surface. The convective heat flux is composed of convection due to the translational and rotational modes, as well as due to the vibrational mode. The convective heat flux is modeled according to Fourier's law, and species diffusion heat fluxes are modeled using a modified form of Fick's law [39].

A radiative equilibrium boundary condition, as shown in Eq. (16), is used for the baseline results and part of the sensitivity study. The wall temperature $T_{w}$ is set by this boundary condition with emissivity $\epsilon$ for graphite set to 0.83 [15]:

$$
\boldsymbol{q}_{\mathrm{conv}}+\boldsymbol{q}_{\mathrm{diff}}=\sigma \varepsilon T_{w}^{4}
$$

The contribution of conductive heat transfer is also evaluated by using the material response code MOPAR developed at the University of Michigan $[40, \underline{41}]$. MOPAR is coupled to LeMANS and can model heat conduction within the material. The energy balance at the surface is calculated using the boundary condition shown in Eq. (17):

$$
\boldsymbol{q}_{\mathrm{cond}}=\boldsymbol{q}_{\mathrm{conv}}+\boldsymbol{q}_{\mathrm{diff}}-\epsilon \sigma\left(T_{w}^{4}-T_{\mathrm{res}}^{4}\right)
$$

where $T_{\text {res }}$ is the constant reservoir temperature. The coupled simulations begin with the converged flowfield solution obtained from LeMANS. LeMANS first calls MOPAR, and an initial $\boldsymbol{q}_{\text {cond }}$ is calculated based on the total heat flux from LeMANS (i.e., initial heat flux) from Eq. (17). MOPAR then runs for a user-defined time, and the wall temperature is calculated. MOPAR passes this wall temperature value to LeMANS, and then the fluid equations are solved for a user-defined number of iterations. The temperature along the wall remains constant during this computation. MOPAR is then called again, and the updated value along with the initial value of conductive heat flux are used as temporal boundary conditions to solve the time- 
Table 2 Freestream and wall boundary conditions

\begin{tabular}{lc}
\hline \hline Parameter & Value \\
\hline Mass flow rate, $\mathrm{kg} / \mathrm{s}$ & $0.82 \times 10^{-3}$ \\
Velocity, $\mathrm{m} / \mathrm{s}$ & 136 \\
Temperature $T_{\infty}, \mathrm{K}$ & 7000 \\
Pressure, $\mathrm{kPa}$ & 21.3 \\
Wall temperature $T_{\text {wall }}, \mathrm{K}$ & 1598 \\
\hline \hline
\end{tabular}

accurate energy equation. The process is repeated until a converged steady-state solution is obtained. In this study, the criterion for convergence is when the wall temperature values are the same between final and previous calls of MOPAR.

\section{Numerical Setup}

The test conditions [i.e., facility inlet (ICP torch exit) conditions] and the graphite sample wall temperature simulated by LeMANS are based on the experiments conducted at the University of Vermont in order to compare the computational results with the experimental measurements. The simulations are performed for the experimental conditions that are shown in Table 2. The boundary conditions assigned for the simulations are shown in Fig. 2. An axisymmetric configuration is used for all simulations. The test chamber wall is set as a noncatalytic wall with an isothermal wall temperature of $300 \mathrm{~K}$. The test sample wall is set to a radiative equilibrium boundary condition, and the FRSC model is applied only to this wall. In addition, the stagnation point is also shown where the heat flux and the surface temperature are measured. The grid is generated using the commercial mesh generation software Pointwise [42]. Grid independence is achieved for a flow in thermal equilibrium using a grid with 22,000 quadrilateral cells. A picture of the grid is shown in Fig. 3 .

The gas-surface interaction processes studied are the recombination of nitrogen atoms to molecules at the surface due to catalysis and carbon nitridation where nitrogen atoms react with the surface carbon to form gaseous CN. Carbon nitridation is studied as sample mass loss is observed in the experiment, and results for the surface recession are reported in the work by Lutz et al. [9,43] Therefore, two sets of surface reactions are taken into account using the FRSC model. The first set is the surface reaction [shown in Eq. (18)] that accounts only for the nitrogen atom recombination on the wall due to surface catalysis. Here, a gaseous nitrogen atom is adsorbed onto an available active site on the surface through an adsorption reaction. Then, another nitrogen atom from the gas phase recombines with the adsorbed nitrogen atom to form a gaseous nitrogen molecule and leaves the active site. In these reactions, $k_{f 1}$ and $k_{f 2}$ are the respective forward reaction rates:
Adsorption $\left(E_{\mathrm{ad}}=0 \mathrm{~J} / \mathrm{mol}\right)$

$$
\mathrm{N}+(s) \stackrel{k_{f 1}}{\rightarrow} \mathrm{N}(s)
$$

Eley-Rideal recombination $\left(E_{\mathrm{ER}}=0 \mathrm{~J} / \mathrm{mol}\right)$

$$
\mathrm{N}+\mathrm{N}(s) \stackrel{k_{f 2}}{\rightarrow} \mathrm{N}_{2}+(s)
$$

The second set of surface reactions [shown in Eq. (19)] takes into account the nitrogen atom recombination on the wall due to surface catalysis along with the carbon nitridation reaction where the carbon from the surface reacts with the impinging nitrogen atoms. The EleyRideal recombination reaction is used to represent the process of carbon nitridation. In these reactions, $k_{f 1}, k_{f 2}$, and $k_{f 3}$ are the respective forward reaction rates:

Adsorption $\left(E_{\mathrm{ad}}=0 \mathrm{~J} / \mathrm{mol}\right)$

$$
\mathrm{N}+(s) \stackrel{k_{f 1}}{\rightarrow} \mathrm{N}(s)
$$

Eley-Ridel recombination $\left(E_{\mathrm{ad}}=0 \mathrm{~J} / \mathrm{mol}\right)$

$$
\mathrm{N}+\mathrm{N}(s) \stackrel{k_{f 2}}{\rightarrow} \mathrm{N}_{2}+(s)
$$

Eley-Ridel recombination $\left(E_{\mathrm{ad}}=0 \mathrm{~J} / \mathrm{mol}\right)$

$$
\mathrm{N}+(s)+C_{b} \stackrel{k_{f 3}}{\rightarrow} \mathrm{CN}+(s)
$$

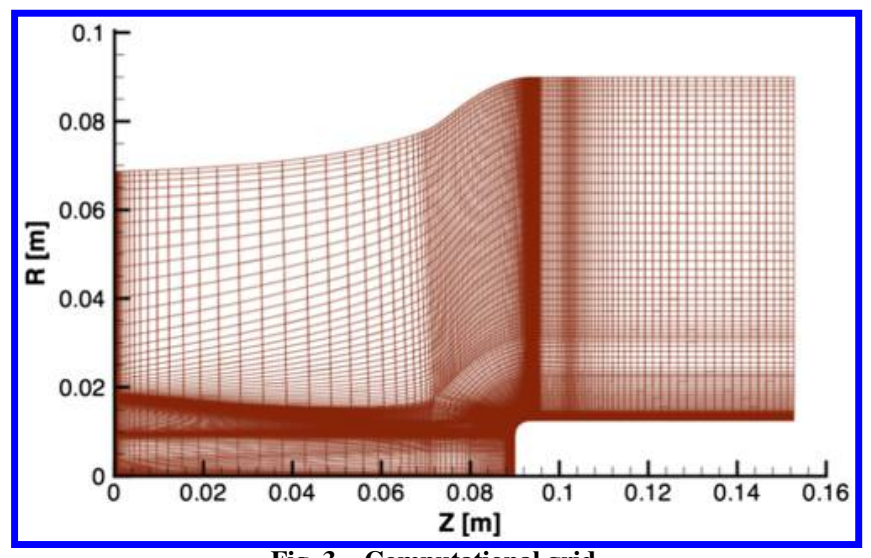

Fig. 3 Computational grid.

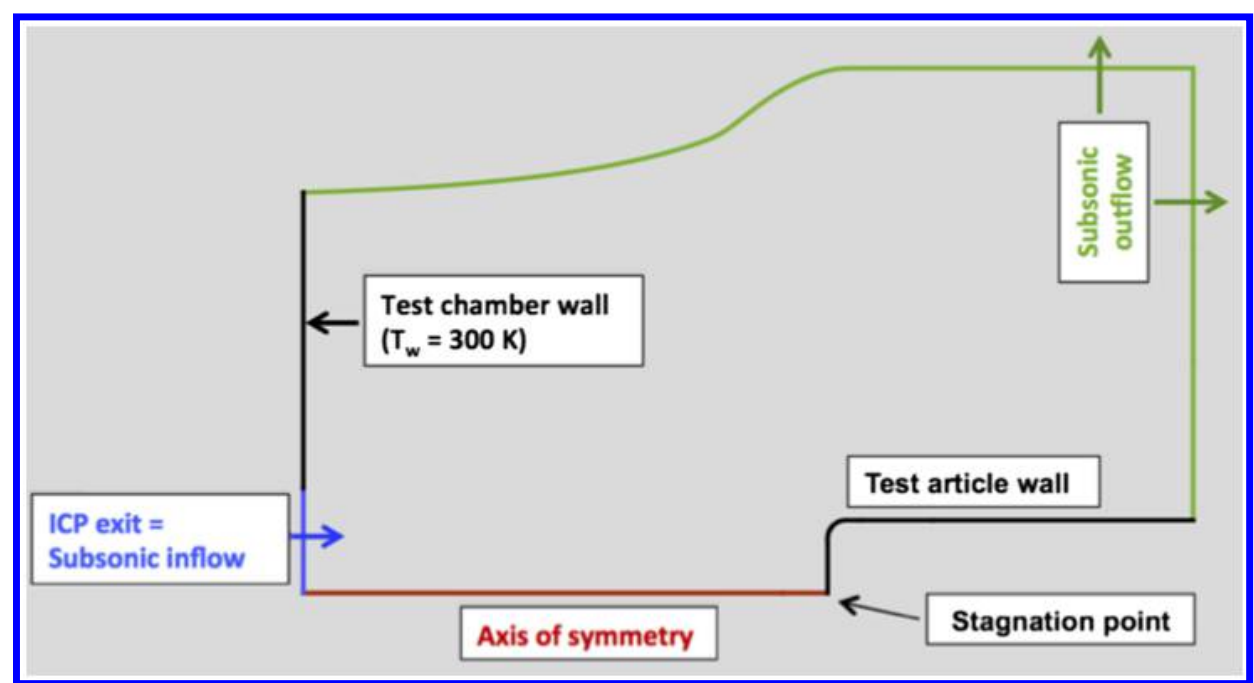


All the test cases are investigated using a constant reaction efficiency $\gamma$. The effective reaction efficiency for a gas-phase reactant consumed in a surface reaction process is the net result of competing finite rate processes. An analytic expression for constant reaction efficiency $\gamma$ is derived for both the surface reaction set shown in Eq. (18) as well as for Eq. (19).

The constant reaction efficiency $\gamma$ for a gas-phase reactant $k$ is defined as the fraction of collisions that it experiences, with the surface resulting in its loss from the gas-phase environment:

$$
\gamma=\frac{-\dot{w}_{k}}{\Gamma_{k}}
$$

where $\dot{w}_{k}$ is given by Eq. (4), and $\Gamma_{k}$ is the impingement flux given by Eq. (8). The negative production rate $\dot{w}_{k}$ represents the consumption of the gas-phase reactant at the wall. The constant reaction efficiency $\gamma$ for the surface reactions in Eq. (18) can be calculated using Eq. (4), Eq. (ㅁ), and Eq. ( $\underline{8})$ as

$$
\gamma=\frac{-\dot{w}_{N}}{\Gamma_{N}} \quad \gamma=\frac{k_{f 1} C_{N} \Phi_{s, e}+k_{f 2} C_{N} \Phi_{s, N}}{\left(\bar{\nu}_{N} / 4\right) C_{N}}
$$

The surface concentration of the adsorbed $\mathrm{N}$ atoms is obtained by equating Eq. (4) to zero for steady-state conditions as

$$
\dot{w}_{N(s)}=0 \quad k_{f 1} C_{N} \Phi_{s, e}-k_{f 2} C_{N} \Phi_{s, N}=0 \quad \Phi_{s}=\Phi_{s, e}+\Phi_{s, N}
$$

Substituting for $k_{f 1}$ and $k_{f 2}$ from Eqs. (9) and (11),

$$
\Rightarrow \Phi_{s, N}=\frac{\Phi_{s} S_{0}}{S_{0}+\gamma_{0}}
$$

Solving Eqs. (21) and (22), the net constant reaction efficiency $\gamma$ is given by

$$
\gamma=\frac{2 S_{0} \gamma_{0}}{S_{0}+\gamma_{0}}
$$

The constant reaction efficiency $\gamma$ for the surface reactions in

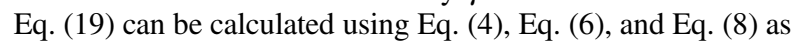

$$
\gamma=\frac{k_{f 1} C_{N} \Phi_{s, e}+k_{f 2} C_{N} \Phi_{s, N}+k_{f 3} C_{N} \Phi_{s, e} \chi_{b 1, c}}{\left(\bar{\nu}_{N} / 4\right) C_{N}}
$$

The bulk phase surface concentration $\chi_{b 1, c}$ is one for a single bulk phase. Solving Eqs. (24) and (22), the net constant reaction efficiency $\gamma$ is given by

$$
\gamma=\frac{2 S_{0} \gamma_{0}+\gamma_{\mathrm{CN}} \gamma_{0}}{S_{0}+\gamma_{0}}
$$

The reaction efficiency $\gamma_{0}$ for surface catalysis, also referred to as catalytic efficiency of nitrogen atoms, is denoted by $\gamma_{\mathrm{N}}$. It is defined as the ratio of the flux of nitrogen atoms that recombine on the surface to form nitrogen molecules to the total flux of nitrogen atoms that impinge on the surface. A constant catalytic efficiency is achieved by setting $S_{0}$ equal to $\gamma_{0}$. The reaction efficiency for carbon nitridation, also referred to as carbon nitridation efficiency, is denoted by $\gamma_{\mathrm{CN}}$. It is defined as the ratio of nitrogen atoms reaching the surface and combining with surface carbon atoms to the ratio of the total flux of nitrogen atoms that impinge on the surface. It is assumed in this investigation that all the carbon mass loss occurs due to the carbon nitridation reaction.

The following dissociation-recombination reactions are considered in the analysis:

$$
\mathrm{N}_{2}+\mathrm{M} \rightleftharpoons 2 \mathrm{~N}+\mathrm{M}
$$

$$
\mathrm{CN}+\mathrm{M} \rightleftharpoons \mathrm{C}+\mathrm{N}+\mathrm{M} \quad \mathrm{M}=\mathrm{N}, \mathrm{N}_{2}, \mathrm{C}, \mathrm{CN}
$$

Table 3 Test cases

\begin{tabular}{lccc}
\hline \hline Case & $\begin{array}{c}\text { Catalytic } \\
\text { efficiency } \gamma_{\mathrm{N}}\end{array}$ & $\begin{array}{c}\text { Carbon nitridation } \\
\text { efficiency } \gamma_{\mathrm{CN}}\end{array}$ & $\begin{array}{c}\text { Effective reaction } \\
\text { efficiency } \gamma\end{array}$ \\
\hline 2 & 0 & 0 & 0 \\
2 & 0.07 & 0 & 0.07 \\
3 & 0.07 & 0.005 & 0.0725 \\
4 & 1 & 0 & 1 \\
\hline \hline
\end{tabular}

For the analysis where only catalytic nitrogen atom recombination at the surface is considered, the gas mixture is composed of atomic and molecular nitrogen, and only Eq. (26) is used. For the analysis where both catalytic nitrogen atom recombination and carbon nitridation reaction at the surface is considered, the gas mixture is composed of atomic nitrogen, molecular nitrogen, atomic carbon, and the $\mathrm{CN}$ molecule, and it uses both Eqs. (26) and (27).

The test cases considered in this study to determine the effects of gas-surface interaction processes are shown in Table 3 . The catalytic efficiency of nitrogen atoms $\gamma_{\mathrm{N}}$ is set to zero for a noncatalytic wall and is set to one for a fully catalytic wall. The partially catalytic wall condition of $\gamma_{\mathrm{N}}=0.07$ is based on an experimentally determined value [44] for pure carbon. The value for carbon nitridation efficiency $\gamma_{\mathrm{CN}}$ is set equal to 0.005 based on a value determined by Driver and MacLean [45]. It is obtained from a comparison between data from arcjet tests performed for phenolic-impregnated carbon ablator in nitrogen and results from computational simulations. It should be noted that $\gamma_{\mathrm{CN}}$ is dependent on the type of carbon used as well as the experimental conditions.

Case 1 represents a wall where no surface chemistry is accounted for and is treated as noncatalytic. The surface chemistry for cases 2 and 4 is defined by the reactions shown in Eq. (18) and, for case 3, it is defined by Eq. (19). The effective reaction efficiency $\gamma$ for cases 2 and 4 is calculated using Eq. (23) and, for case 3, Eq. (25) is used.

\section{Results}

The comparisons of computational results with the measured experimental data are presented here. The flow physics model chosen for all test cases is thermochemical nonequilibrium flow. Thermochemical nonequilibrium means that both vibrational relaxation and the finite rate of chemical relaxation are considered. The translational and rotational modes are assumed to be equilibrated. It is shown in [18] that flow is in thermochemical nonequilibrium for the flowfield conditions. A uniform velocity, temperature, and density profile at the facility inlet (i.e., the quartz tube exit) is specified in the simulations. The flow at the inlet of the test chamber is nonuniform. Therefore, a study is performed to assess the effects of different inlet profiles (i.e., uniform and nonuniform) on the species concentration and temperature gradients near the material surface, as well as on the heat transfer to the material surface. It is concluded that the nonuniform inlet profile does not significantly affect the solution. The results for the study are discussed in $[18, \underline{46}]$. An assumption of chemical equilibrium of the nitrogen gas mixture at the ICP torch exit is used to calculate its composition in these simulations, as experimental data at the exit are not available. The equilibrium composition of the nitrogen gas mixture at the quartz tube exit for the given temperature and pressure [9] is calculated using the NASA program Chemical Equilibrium with Applications (CEA) [47].

The main calculated parameters analyzed are translational temperature, normalized nitrogen atom density, surface heat flux, and mass removal rate. The normalized nitrogen atom density is calculated by scaling the nitrogen atom density value for each case with the respective value at the location of the experimentally measured data at the largest distance from the stagnation point of the test sample along the stagnation line. The experimental temperature and normalized nitrogen atom density values have uncertainties of about $\pm 500 \mathrm{~K}$ and $\pm 25 \%$, respectively [48]. The simulation runtime for each case is approximately $6 \mathrm{~h}$ using 32 processors.

In Sec. V.A, the baseline results for the comparisons of computational results with the measured experimental data are presented. It 

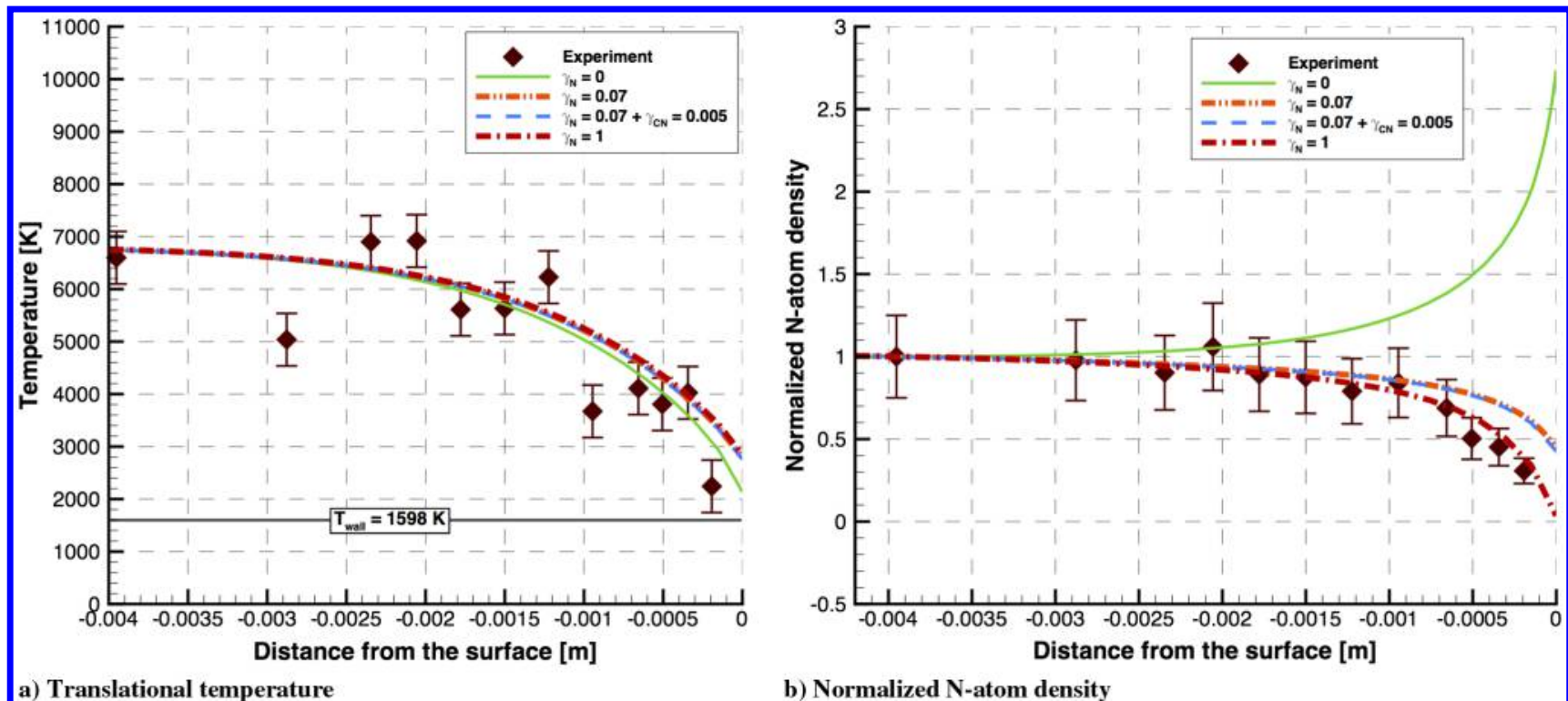

Fig. 4 Comparison of translational temperature and normalized $\mathrm{N}$-atom density along the stagnation line.
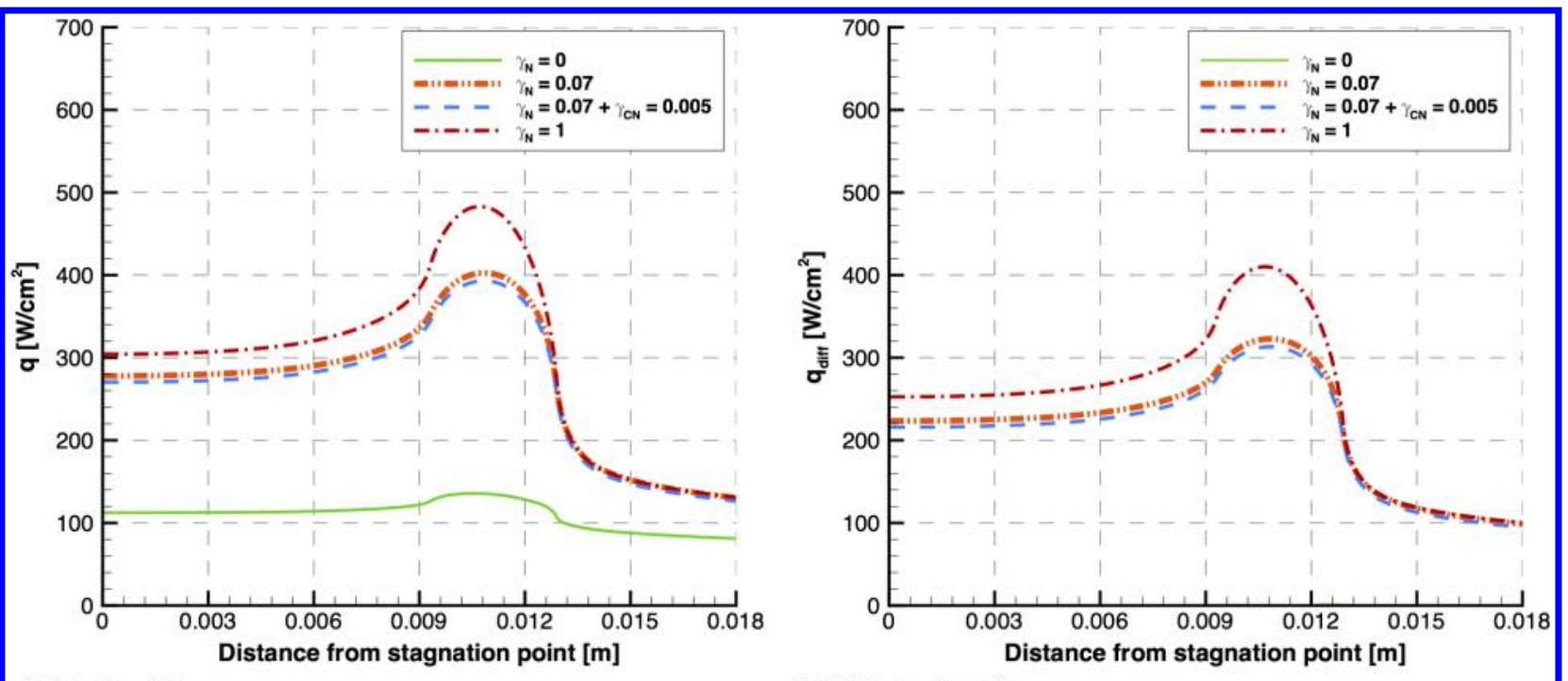

a) Total heat flux

b) Diffusive heat flux

Fig. 5 Comparison of wall heat flux between the computational results.

is seen in the baseline results that there are significant discrepancies between the computational and experimentally measured values, which could be explained by a combined effect of various mechanisms that are addressed in this study. Section V.B presents the results of the sensitivity analysis performed on the ICP torch exit chemical composition to evaluate its effects on the flow parameters in the boundary layer and the surface properties and their comparison with the measured experimental data. The results of the study performed to determine the effects of conduction within the graphite sample on the surface properties are also presented in Sec. V.C.

\section{A. Baseline Results}

The comparisons between the numerical results and experimental LIF measurements are presented for translational temperature and normalized nitrogen atom density in the test sample boundary layer along the stagnation streamline. The stagnation line boundary-layer results are shown for the translational temperature in Fig. $4 \mathrm{a}$ and the normalized nitrogen atom density in Fig. 4b. There is a rise in temperature (Fig. $4 \mathrm{a}$ ) in the boundary layer for cases where surface chemistry is included as compared to case 1 for a noncatalytic wall. The comparisons between cases 2, 3, and 4 show that temperature in the boundary layer are not significantly affected for different surface- chemistry processes. There is an increase in nitrogen atom density (Fig. 4b) in the boundary layer for case 1, whereas it is consumed for all other cases due to surface chemical reactions. The nitrogen atom loss is due to surface catalysis, i.e., catalytic recombination of nitrogen atoms to molecules for cases 2 and 4 . The nitrogen atom loss seen for case 3 is the combined effect of surface catalysis as well as carbon nitridation. The nitrogen atom density for all cases except case 1 show a loss of nitrogen atoms in the boundary layer, as is observed in the experimental measurements.

The effect of surface chemistry on the surface properties is also evaluated. The properties analyzed are the surface heat flux and wall temperature. The total heat flux is plotted in Fig. 5a along with the diffusive heat flux in Fig. 5b. An increase is seen in the total heat flux for all the cases with surface reactions as compared to the noncatalytic wall. This increase is explained by the contribution from diffusive heat flux for the cases with surface reactions, which is zero for a noncatalytic wall.

The wall temperature for results from different surface-chemistry models is shown in Fig. 6a. Case 4 with a fully catalytic wall has the highest, and case 1 with no surface chemistry has the lowest temperature at the surface. The carbon mass removal flux $\dot{m}_{b}$ as a result of the carbon nitridation reaction for case 3 is also computed and is 


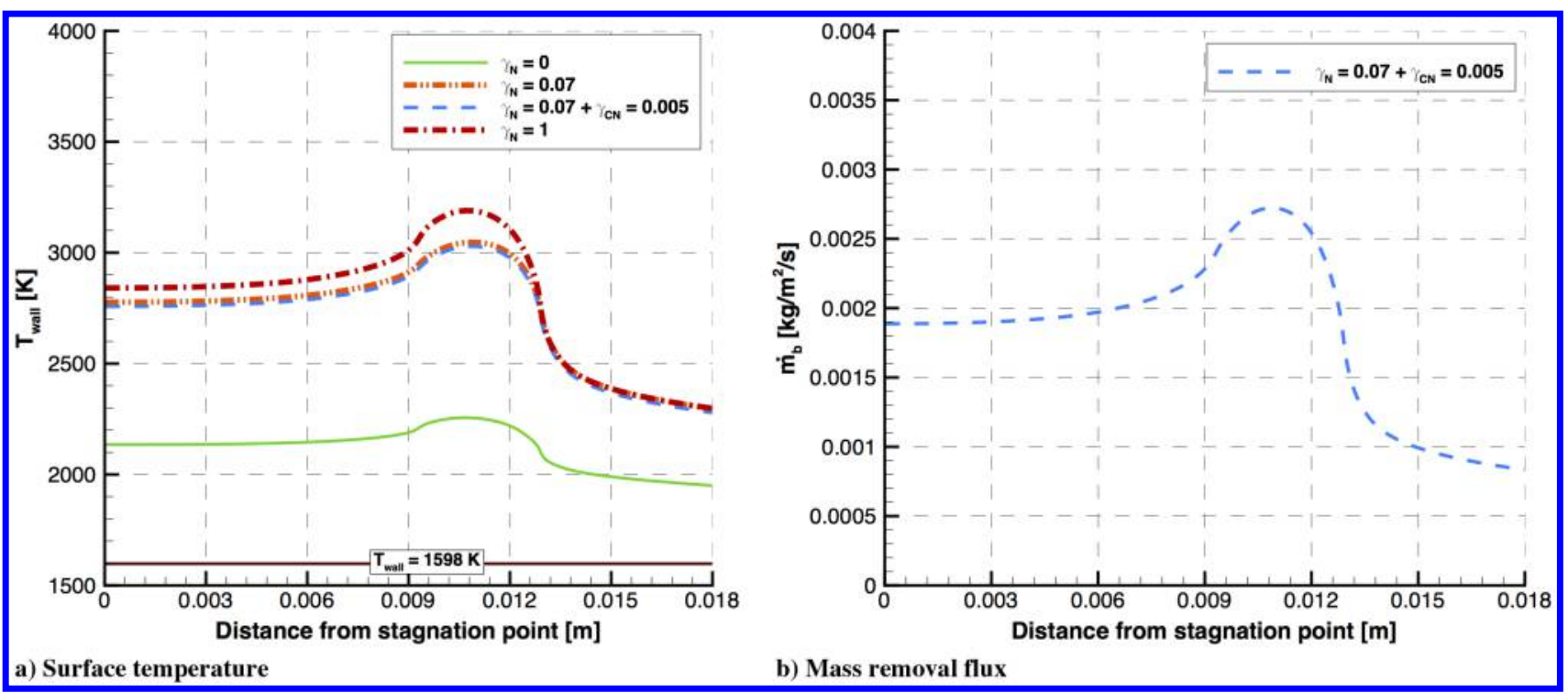

Fig. 6 Comparison of temperature for different cases and mass removal flux for case 3.

shown in Fig. 6b. The total mass loss rate is calculated from $\dot{m}_{b}$, as shown in Eq. $(\underline{28})$ :

$$
\text { mass loss rate }=\int \dot{m}_{b} \mathrm{~d} A
$$

where $\dot{m}_{b}$ is the mass removal flux [calculated using Eq. (15)] for each surface element, and $\mathrm{d} A$ is the surface area of each element. The stagnation point heat flux is measured experimentally using a slug calorimeter for test conditions similar to this case. The heat flux is measured for the case of a $0.84 \mathrm{~g} / \mathrm{s}$ mass flow rate and pressure of $21.3 \mathrm{kPa}$. A comparison between the experimental and computed values for stagnation point heat flux, temperature, and mass loss rate for case 3 is provided in Table 4 .

It can be seen that the computational values are much higher than the experimentally measured values. The higher computed values could be attributed to a higher degree of nitrogen atom flux to the surface in the calculations. An assumption of chemical equilibrium of the nitrogen gas mixture at the ICP torch exit is used in the baseline simulations. The species present in the gas mixture are molecular and atomic nitrogen with mole fractions of 0.15 and 0.85 , respectively. The equilibrium gas mixture composition is probably more dissociated than the composition for a chemically reacting flow with finite rate chemistry. Therefore, the flux to the test sample of dissociated nitrogen atoms available might be less than used in these simulations.

A lower nitrogen atom flux to the surface would result in a lower mass removal rate. A sensitivity analysis is performed on the ICP torch exit chemical composition to evaluate its effects on the flow parameters in the boundary layer and the surface properties. The analysis is performed for case 3 to include the effects of the inlet gas composition on carbon mass removal. The results of the sensitivity analysis performed on the ICP torch exit chemical composition are presented Sec. V.B. A radiative equilibrium boundary condition is used in these simulations where the heat conduction within the sample is not included that, if accounted for, may affect the surface properties. Section V.C presents the results of the study performed to determine the effects of conduction within the sample.

Table 4 Stagnation point values and mass loss rates

\begin{tabular}{lccc}
\hline \hline & $q_{\text {stag }}, \mathrm{W} / \mathrm{cm}^{2}$ & $T_{\text {stag }}, \mathrm{K}$ & Mass loss rate, $\mathrm{mg} / \mathrm{s}$ \\
\hline CEA & 270 & 2757 & 2.2 \\
Experiment & $40-80$ & $\sim 1600$ & $0.2-0.6$ \\
\hline \hline
\end{tabular}

\section{B. Sensitivity to Inlet Chemical Composition}

1. Comparison Between Equilibrium and Power Equal to 13.8 Kilowatts

This section presents the comparison performed between the results obtained using the inlet gas composition calculated assuming chemical equilibrium and that obtained for a ICP torch flow power. The power absorbed by the flow in the ICP torch is used to determine the composition of the gas at the test chamber inlet, i.e., the ICP torch exit. The power in the flow is the product of the voltage, current, and an efficiency factor estimated to be $0.56 . \underline{\S}$ The voltage is $10.3 \mathrm{kV}$ and the current is $2.4 \mathrm{~A}$ current. The calculated power is $13.8 \mathrm{~kW}$. The flow power is also given by the product of the mass flow rate $\dot{m}_{\text {flow }}$ and specific enthalpy $h$ given by Eq. (29). The specific enthalpy is dependent on the composition of the mixture given by the mass fraction of the gas mixture species $Y_{i}$ and the inlet temperature of the flow $T$ :

$$
\begin{aligned}
& \text { Power }=\dot{m}_{\mathrm{flow}} \Delta h \\
& \Delta h=\sum_{i=N, N_{2}} Y_{i} \int_{298}^{T} C_{p i} \mathrm{~d} T+\sum_{i=N, N_{2}} Y_{i} \Delta h_{f i}
\end{aligned}
$$

where

$$
\begin{aligned}
& Y_{i}=\frac{M_{i}}{M_{\mathrm{avg}}} X_{i} ; \quad M_{\mathrm{avg}}=\sum_{i=N, N_{2}} X_{i} M_{i} \\
& C_{p i}=C_{v i}+R_{i} \\
& C_{v i}=C_{v i, t}+C_{v i, r}+C_{v i, \mathrm{vib}}+C_{v i, \mathrm{el}} \\
& C_{v i, t}=1.5 R_{i}
\end{aligned}
$$

For $i=N_{2}$

$$
C_{v i, r}=R_{i} ; \quad C_{v i, \mathrm{vib}}=R_{i} \frac{\left(\theta_{\mathrm{vib}, i} / T_{\mathrm{vib}, i}\right)^{2} \exp \left(\theta_{\mathrm{vib}, i} / T_{\mathrm{vib}}\right)}{\left[\exp \left(\theta_{\mathrm{vib}, i} / T_{\mathrm{vib}}\right)-1\right]^{2}}
$$

For $i=N$

$$
C_{v i, r}=0 ; \quad C_{v i, \mathrm{vib}}=0
$$

For $i=N, N_{2}$,

${ }^{\S}$ Personal conversation with W. Owens, University of Vermont, Mechanical Engineering, Burlington,VT, January 2013. 


$$
C_{v i, \mathrm{el}}=R_{i}\left\{\frac{\sum_{j=1}^{\infty} g_{j, i}\left(\theta_{\mathrm{el}, j, i} / T_{\mathrm{el}}\right)^{2} \exp \left(-\theta_{\mathrm{el}, j, i} / T_{\mathrm{el}}\right)}{\sum_{j=0}^{\infty} g_{j, i} \exp \left(-\theta_{\mathrm{el}, j, i} / T_{\mathrm{el}}\right)}-\frac{\left[\sum_{j=1}^{\infty} g_{j, i} \theta_{\mathrm{el}, j, i} \exp \left(-\theta_{\mathrm{el}, j, i} / T_{\mathrm{el}}\right)\right]\left[\sum_{j=0}^{\infty} g_{j, i}\left(\theta_{\mathrm{el}, j, i} / T_{\mathrm{el}}^{2}\right) \exp \left(-\theta_{\mathrm{el}, j, i} / T_{\mathrm{el}}\right)\right]}{\left[\sum_{j=0}^{\infty} g_{j, i} \exp \left(-\theta_{\mathrm{el}, j, i} / T_{\mathrm{el}}\right)\right]^{2}}\right\}
$$

where $X_{i}$ is the species mole fraction; and $M_{i}$ and $M_{\text {avg }}$ are the individual species and average gas mixture molecular weight, respectively. $C_{p i}$ is the species-specific heat at constant pressure, $R_{i}$ is the species gas constant, and $h_{f i}$ is the species heat of formation. $C_{v i}$ is the species-specific heat at constant volume. The subscripts in $C_{v i, t}$, $C_{v i, r}, C_{v i, \text { vib }}$, and $C_{v i, \mathrm{el}}$ represent the translational, rotational, vibrational, and electronic specific heats at constant volume, respectively. $\theta_{\mathrm{vib}, i}$ is the species characteristic vibrational temperature. $\theta_{\mathrm{el}, j, i}$ and $g_{j, i}$ are the characteristic electronic temperature and the degeneracy of the $j$ th energy level, respectively [이].

The composition of the gas mixture is calculated for $13.8 \mathrm{~kW}$ power using the mass flow rate and inlet temperature given in Table 2 . The power in the flow for chemical equilibrium composition at inlet and $7000 \mathrm{~K}$ inlet temperature corresponds to $30 \mathrm{~kW}$, which is $100 \%$ higher than the calculated power.

The relation of power with the gas mixture composition is shown in Fig. 7, where power is plotted against the nitrogen atom mole fraction $X_{\mathrm{N}}$ for a constant temperature of $7000 \mathrm{~K}$ [calculated using Eq. (29)]

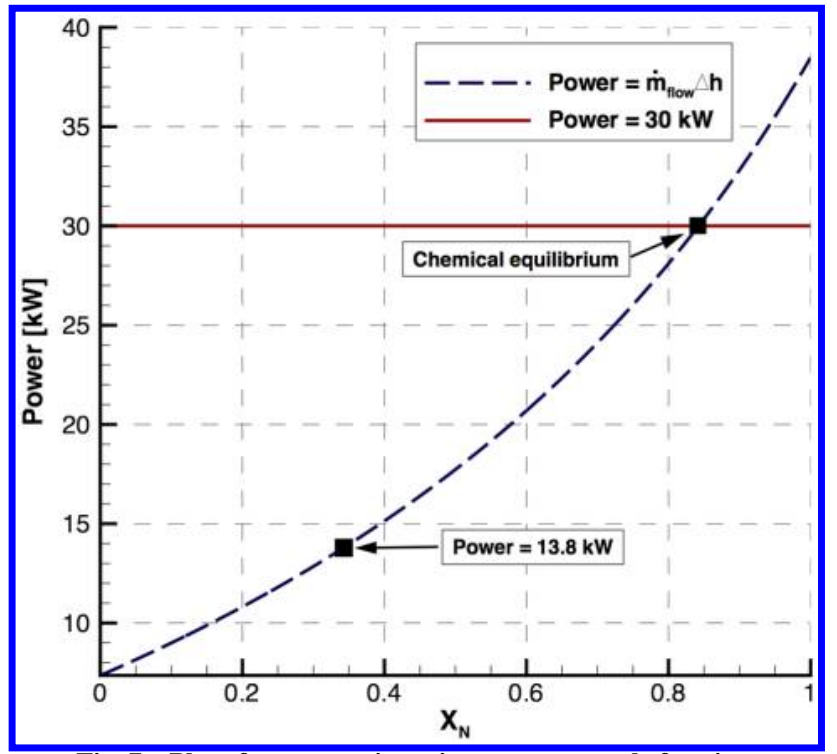

Fig. 7 Plot of power against nitrogen atom mole fraction. along with the constant power of $30 \mathrm{~kW}$ in the flow for chemical equilibrium composition at the inlet. It can be seen that the power in the flow is minimum for the zero nitrogen atom mole fraction, i.e., the flow is not dissociated. The flow power increases with the level of dissociation in the flow and is maximum for fully dissociated flow $\left(X_{\mathrm{N}}=1\right)$. The higher the degree of dissociation, the higher the power.

Based on the chemical equilibrium and $13.8 \mathrm{~kW}$ inlet compositions, the translational temperature and normalized nitrogen atom number density profiles along the stagnation line in the boundary layer are shown in Figs. 8a and $\underline{8 b}$, respectively. The translational temperature in the boundary layer for $13.8 \mathrm{~kW}$ power is lower in comparison to the equilibrium inlet composition. The reason for this is that the temperature in the flow decreases with a decrease in enthalpy. Enthalpy is directly related to power [Eq. (29)]. Lower power in the flow leads to lower temperature. Further discussion on the effect of varying temperature and power in the flow is presented in Secs. II and III. The root-mean-square percentage error between the translational temperature for the chemical equilibrium inlet composition and the experimental values is $16.1 \%$. The root-mean-square percentage error between the translational temperature for $13.8 \mathrm{~kW}$ power and the experimental values is $16.3 \%$.

The equilibrium inlet composition has a higher normalized nitrogen atom number density in the boundary layer in comparison to the inlet composition calculated using $13.8 \mathrm{~kW}$ power. The root-meansquare percentage error between the normalized nitrogen atom number density for the chemical equilibrium inlet composition and the experimental values is $15.2 \%$. The root-mean-square percentage error between the normalized nitrogen atom number density for $13.8 \mathrm{~kW}$ power and the experimental values is $10.9 \%$. The level of dissociation is higher for chemical equilibrium inlet composition as compared to the $13.8 \mathrm{~kW}$ power inlet composition. Therefore, the nitrogen atom density is higher for the chemical equilibrium case as compared to the $13.8 \mathrm{~kW}$ power case.

The total heat flux and temperature at the wall for these cases are shown in Figs. $\underline{9 a}$ and $\underline{9 b}$, respectively. The mass removal flux is shown in Fig. 10 .

As can be seen, there is a significant reduction in heat flux, the wall temperature, and mass loss for $13.8 \mathrm{~kW}$ power in comparison to the results of equilibrium inlet composition. The comparison between the stagnation point values for the two cases along with the experimental data is shown in Table 5 . There is a $52 \%$ reduction in heat flux,
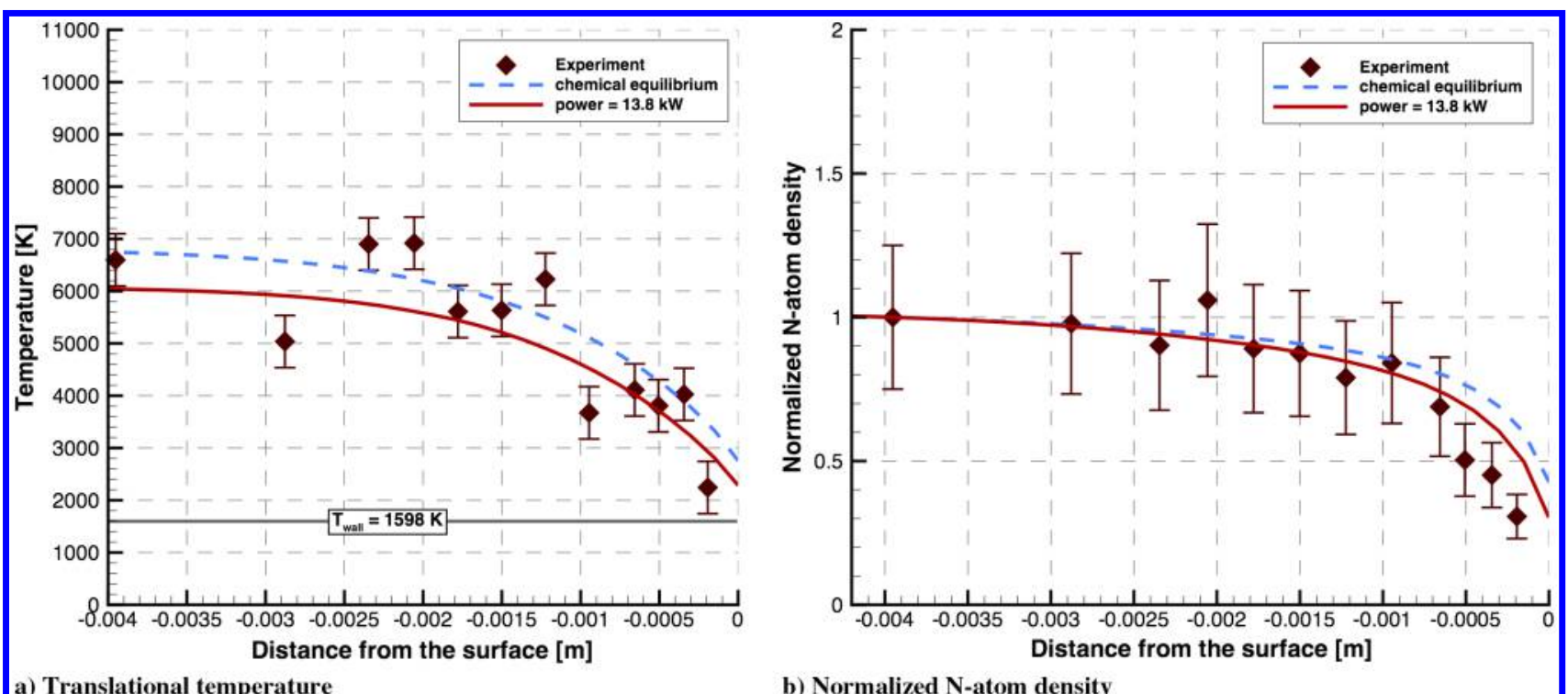

Fig. 8 Comparison of translational temperature and normalized $\mathrm{N}$-atom density along the stagnation line for different inlet compositions. 

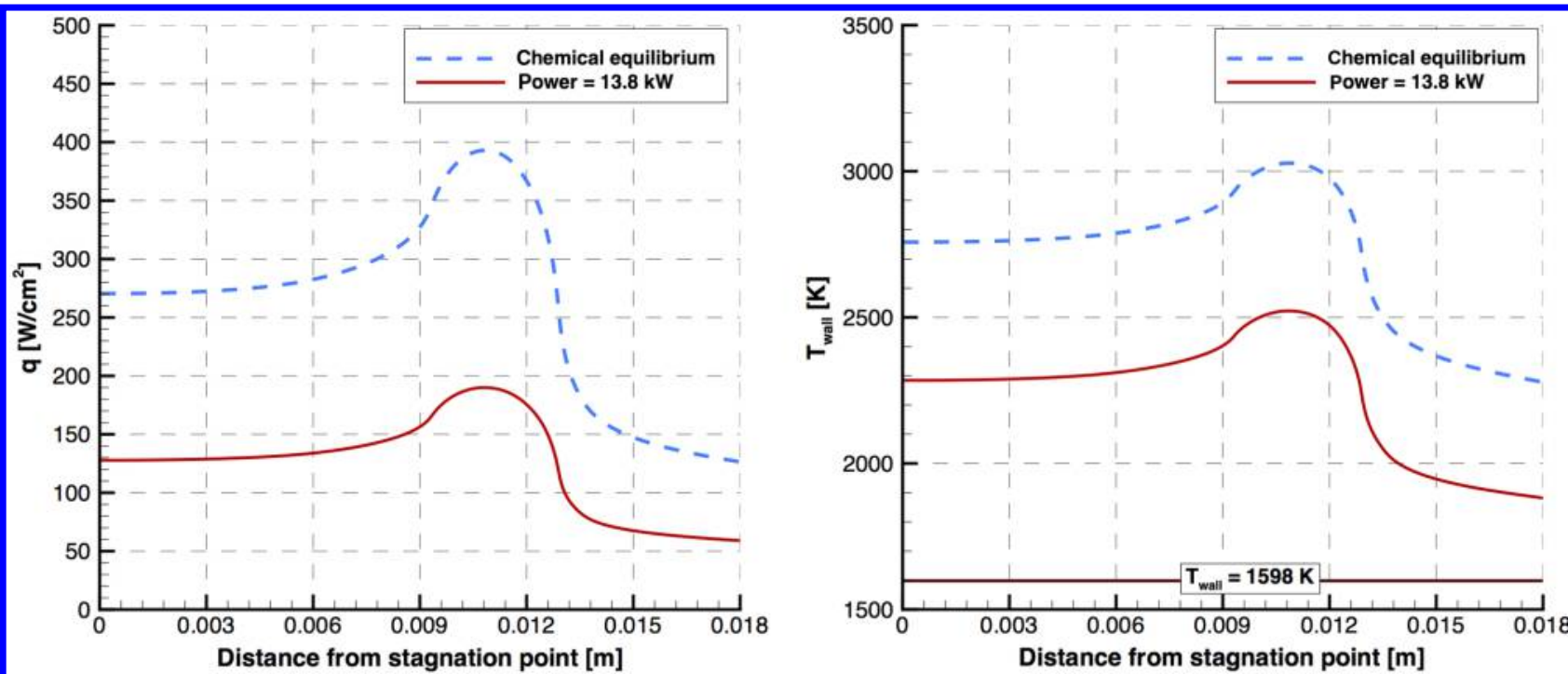

a) Total heat flux

b) Surface temperature

Fig. 9 Comparison of wall heat flux and temperature between the computational results for different inlet compositions.

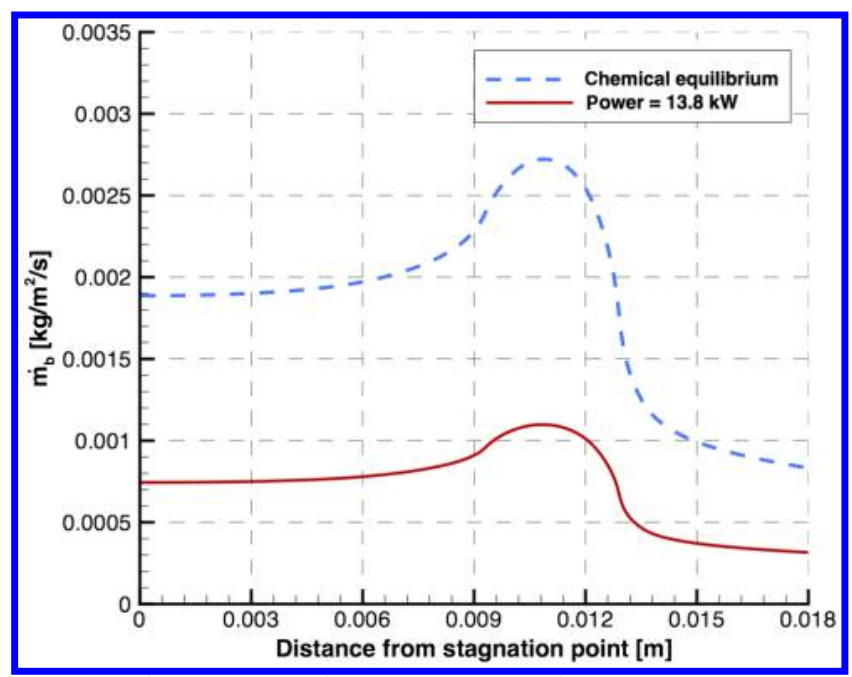

Fig. 10 Comparison of mass removal flux between chemical composition for equilibrium inlet and $13.8 \mathrm{~kW}$ power.

$17 \%$ reduction in wall temperature, and $60 \%$ reduction in mass loss rate for the assumption of $13.8 \mathrm{~kW}$ flow power in comparison with the use of equilibrium chemical composition at the inlet. These values are still higher than the experimentally measured data. The losses in the ICP torch are not quantified experimentally, and hence are not included in the calculation of power (see footnote $\stackrel{\S}{\text { }}$ ). These losses are in addition to the efficiency factor of 0.56 used in the calculation for the power of $13.8 \mathrm{~kW}$. The comparisons suggest that the power in the flow may be even less than $13.8 \mathrm{~kW}$, assuming the rest of the test conditions do not change. Since the ICP torch exit conditions are not well defined, a sensitivity analysis on the ICP torch exit chemical composition is performed for different values of inlet power and temperature. The results of the analysis for sensitivity to inlet temperature are presented in Sec. II; and for inlet power, the results are presented in Sec. III.

Table 5 Stagnation point values and mass loss rates

\begin{tabular}{lcccc}
\hline \hline Inlet & $T_{\text {inf }}, \mathrm{K}$ & $q_{\text {stag }}, \mathrm{W} / \mathrm{cm}^{2}$ & $T_{\text {stag }}, \mathrm{K}$ & Mass loss rate, $\mathrm{mg} / \mathrm{s}$ \\
\hline CEA & 7000 & 270 & 2757 & 2.2 \\
$13.8 \mathrm{~kW}$ & 7000 & 128 & 2284 & 0.86 \\
Experiment & 7000 & $40-80$ & 1600 & $0.2-0.6$ \\
\hline \hline
\end{tabular}

\section{Sensitivity to Inlet Temperature}

The results of the analysis for sensitivity of the flowfield and surface parameters to a varying inlet temperature for constant input power are presented in this section. Three values of inlet temperature (i.e., 6000, 7000, and $8000 \mathrm{~K}$ ) are considered. The chemical composition at the inlet is calculated for a $13.8 \mathrm{~kW}$ power, a mass flow rate, and the respective temperature for each case using Eq. (29). The translational temperature and normalized nitrogen atom number density are shown in Figs. 11a and $11 \mathrm{~b}$, respectively.

It can be seen that there is a negligible effect of varying inlet temperature on the profiles of the translational temperature and the normalized nitrogen atom density. The difference between each respective profile for both the translational temperature and the normalized nitrogen atom density is less than $1 \%$. This negligible effect is best explained by the profiles for translational temperature and absolute nitrogen atom number density along the entire stagnation line, shown in Figs. 12a and 12b, respectively. For example, for the $8000 \mathrm{~K}$ case, as the flow progresses toward the sample, the temperature decreases and the nitrogen atom density increases. All three cases tend to reach a similar mixture composition and temperature as the enthalpy of the flow is constant due to constant power in the flow. One interesting aspect of the inlet flow composition for these cases is the counterintuitive inlet composition. The mole fraction in the gas mixture is a maximum for an inlet temperature of $6000 \mathrm{~K}$ and a minimum for $8000 \mathrm{~K}$. For a higher temperature, the flow is more dissociated as opposed to when it is at a lower temperature. The explanation for this trend is the constant inlet power. For a given inlet power (freestream enthalpy), the level of dissociation and the temperature are related. For a given enthalpy, if the translational temperature is lowered, then the chemical energy (level of dissociation) must increase, and vice versa. To maintain a constant power of $13.8 \mathrm{~kW}$ and respective temperature at the inlet in the flow, enthalpy in the flow is added through the heat of formation of nitrogen atoms. It is achieved by an increase in the density of nitrogen atoms in the flow at the inlet.

The total heat flux and temperature at the wall for these cases are shown in Figs. 13a and 13b, respectively. Both the heat flux and the temperature along the surface increase with an increase in temperature when the power is kept constant. The mass removal flux shows a similar trend and is shown in Fig. 14. The total heat flux, wall temperature, and mass removal flux are highest for the $8000 \mathrm{~K}$ case and lowest for the $6000 \mathrm{~K}$ case. Even though the level of dissociation is lower for the higher temperature at the inlet (Fig. 12b), as the flow progresses toward the test sample, the level of dissociation increases due to a higher temperature. A higher number of atoms diffuse to the surface and recombine (both through catalytic activity and carbon 


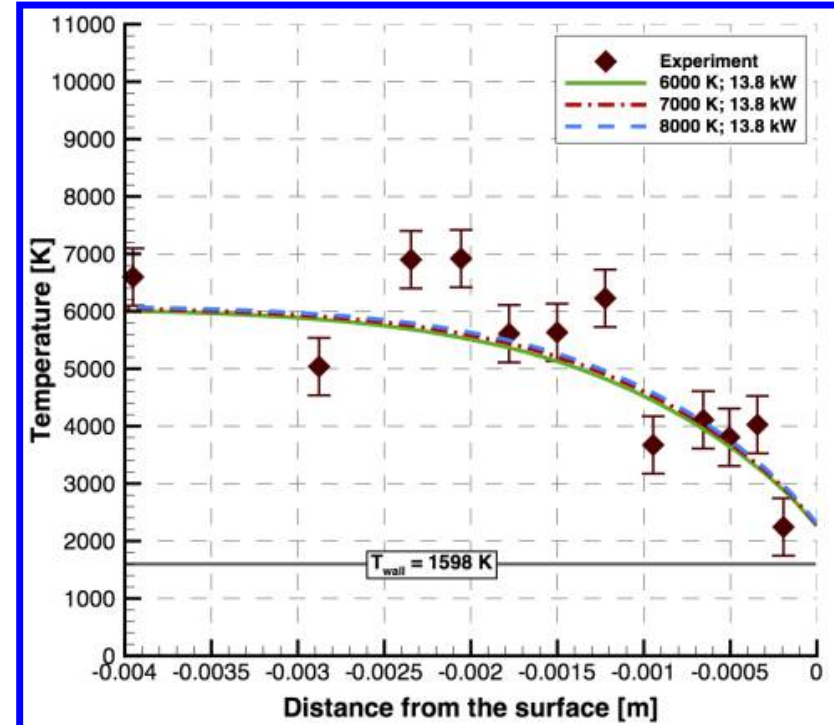

a) Translational temperature

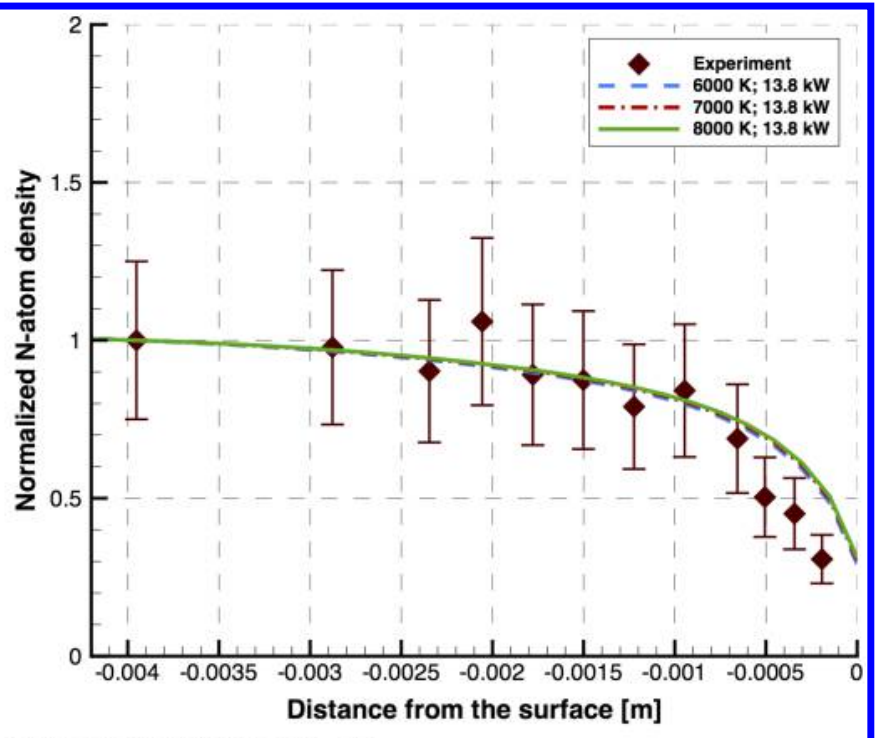

b) Normalized N-atom density

Fig. 11 Comparison of translational temperature and normalized $\mathrm{N}$-atom density along the stagnation line in the boundary layer for varying temperatures.
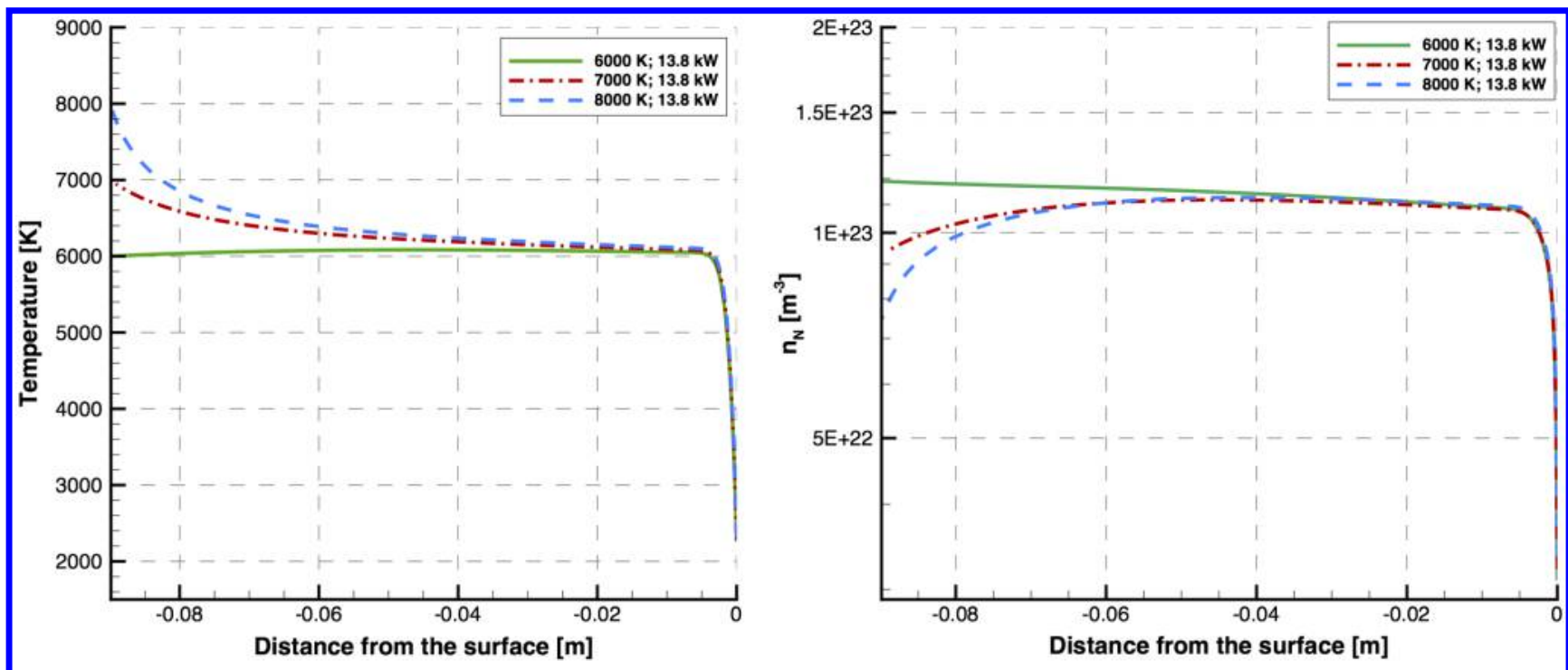

a) Translational temperature

b) $\mathrm{N}$-atom number density

Fig. 12 Comparison of translational temperature and $\mathrm{N}$-atom number density along the stagnation line for varying temperatures.
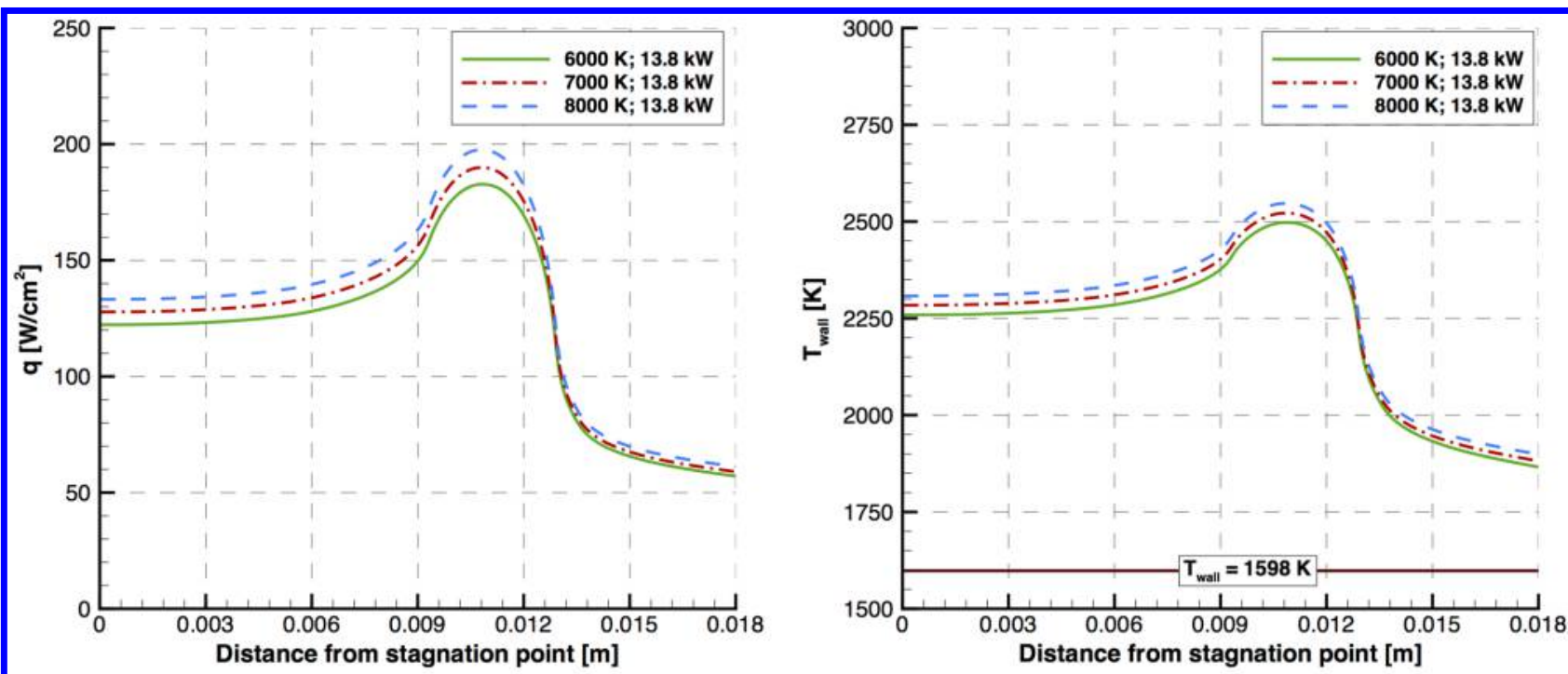

a) Total heat flux

b) Surface temperature

Fig. 13 Comparison of wall heat flux and temperature between the computational results for varying inlet temperatures. 


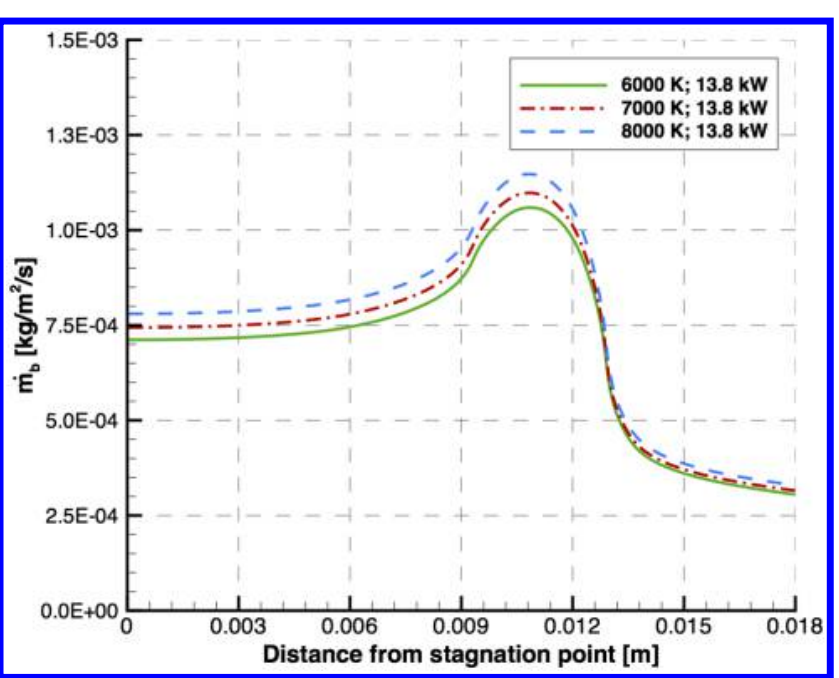

Fig. 14 Comparison of mass removal flux for varying inlet temperature.

nitridation), thus releasing heat of recombination into the surface. This explains the increase in the total heat flux, wall temperature, and mass removal flux along the surface with an increase in inlet temperature.

The comparison between the stagnation point values along with the mass loss rate for these cases is shown in Table $\underline{6}$. The experimental values are also shown in this table. It can be seen that the heat flux, wall temperature, and the mass loss are increased by an increase in inlet temperature. The heat flux is increased by approximately $5 \%$, wall temperature by $1 \%$, and the mass loss by $4 \%$ for every $1000 \mathrm{~K}$ increase in temperature for constant power. The conclusion of this sensitivity study is that the effect of varying the inlet temperature for constant power on translational temperature and the nitrogen atom density in the boundary layer, surface heat flux, wall temperature, and mass loss rate is negligible, and certainly much smaller than the uncertainties in the measurements.

\section{Sensitivity to Input Power}

This section presents the sensitivity of the calculated flowfield and surface parameters to varying input power for a constant inlet temperature. The inlet temperature is $7000 \mathrm{~K}$ for all the cases. This is the experimentally measured value of temperature. Therefore, it is used as the reference temperature to analyze the sensitivity to input power. The power is varied by varying the concentration of nitrogen atoms in the plasma mixture exiting the ICP. The calculated power values along with the respective nitrogen atom mole fraction are

Table 6 Stagnation point values and mass loss rate for varying temperatures

\begin{tabular}{lcccc}
\hline \hline Power, $\mathrm{kW}$ & $T_{\text {inf }}, \mathrm{K}$ & $q_{\text {stag }}, \mathrm{W} / \mathrm{cm}^{2}$ & $T_{\text {stag }}, \mathrm{K}$ & Mass loss rate, $\mathrm{mg} / \mathrm{s}$ \\
\hline 13.8 & 6000 & 122 & 2259 & 0.83 \\
13.8 & 7000 & 128 & 2284 & 0.86 \\
13.8 & 8000 & 133 & 2308 & 0.89 \\
Experiment & 7000 & $40-80$ & 1600 & $0.2-0.6$ \\
\hline \hline
\end{tabular}

Table 7 Stagnation point values and mass loss rate for varying power

\begin{tabular}{lccccl}
\hline \hline Power, $\mathrm{kW}$ & $X_{\mathrm{N}}$ & $T_{\text {inf }}, \mathrm{K}$ & $q_{\text {stag }}, \mathrm{W} / \mathrm{cm}^{2}$ & $T_{\text {stag }}, \mathrm{K}$ & $\begin{array}{l}\text { Mass loss } \\
\text { rate, } \mathrm{mg} / \mathrm{s}\end{array}$ \\
\hline 30 & 0.85 & 7000 & 270 & 2757 & 2.2 \\
13.8 & 0.42 & 7000 & 128 & 2284 & 0.86 \\
10.8 & 0.2 & 7000 & 82 & 2041 & 0.42 \\
9.0 & 0.1 & 7000 & 66 & 1934 & 0.27 \\
7.3 & 0 & 7000 & 52 & 1821 & 0.11 \\
Experiment & -- & 7000 & $40-80$ & 1600 & $0.2-0.6$ \\
\hline \hline
\end{tabular}

shown in Table 7. The efficiency factor is not applied to these power values, as they are dependent on the specified concentration of nitrogen atoms.

The translational temperature profile along the stagnation line in the boundary layer is shown in Fig. 15a. The profiles for 13.8 and $30 \mathrm{~kW}$ (chemical equilibrium) power are also included in the figure. The difference between the translational temperature profiles in the boundary layer is within $1 \%$ for a change in power from 7.3 to $9.0 \mathrm{~kW}$, $2 \%$ for 9.0 to $10.8 \mathrm{~kW}, 5 \%$ for 10.8 to $13.8 \mathrm{~kW}$, and within $13 \%$ for 13.8 to $30 \mathrm{~kW}$. This trend shows that the temperature is sensitive to the change in power. The reason for this is that the temperature in the flow increases with an increase in enthalpy. Enthalpy is directly related to power [Eq. (29)].

The normalized nitrogen atom number density along the stagnation line in the boundary layer is shown in Fig. 15b. The profiles for 13.8 and $30 \mathrm{~kW}$ (chemical equilibrium) power are also included in the figure. The normalized nitrogen atom density is not significantly affected by power. The reason for this is that these profiles are selfnormalized and the trend in the profiles is the same. Also, for all the conditions, the surface chemistry considered is the same. Therefore, the trend in the normalized profile is the same. The case with $30 \mathrm{~kW}$ power shows a difference within $10 \%$ from the $13.8 \mathrm{~kW}$ profile for the region close to the sample. The reason for this is that the $30 \mathrm{~kW}$ case is highly dissociated with a mole fraction of 0.85 at the inlet. This case has the same surface chemistry as the other cases. Therefore, a relatively higher density of nitrogen atoms is in the vicinity of the test sample as compared with the cases with lesser power, and hence lower dissociation.

Even though the normalized nitrogen atom density is not significantly affected by varying power, the absolute nitrogen atom number density is considerably affected, as shown in Fig. 16. There is an approximately $117 \%$ increase in the atom number density in the boundary layer when the power is increased from 7.3 to $9.0 \mathrm{~kW}$ and a $48 \%$ increase for the variation in power from 9.0 to $10.8 \mathrm{~kW}$, a $68 \%$ increase for 10.8 to $13.8 \mathrm{~kW}$, and a $65 \%$ for 13.8 to $30 \mathrm{~kW}$. The reason for the increase in the absolute nitrogen atom number density is the increase in enthalpy in the flow due to increase in power. There is higher energy in the flow that leads to dissociation of nitrogen molecules. The higher the power, the higher the dissociation level.

This shows that the relative values for different test conditions can have similar profiles but the absolute values can vary considerably. It can be concluded from these results that it is very important to obtain absolute measured values for nitrogen atom number densities for validation of computational results with experimental data.

The translational temperature and nitrogen atom number density along the entire stagnation line for varying power and constant inlet temperature are shown in Figs. 17a and 17b, respectively. The sensitivity to power can be seen in both the profiles. The temperature is the highest along the stagnation line for $30 \mathrm{~kW}$ power and is lowest for $7.3 \mathrm{~kW}$ power. This increase in temperature is directly attributed to the increase in enthalpy in the flow due to increase in power in the flow. The nitrogen atom number density is highest along the stagnation line for $30 \mathrm{~kW}$ power and is the lowest for $7.3 \mathrm{~kW}$ power. The higher energy in the flow causes more dissociation. The flow is chemically reacting and, as it progresses, the temperature decreases as the nitrogen atom number density increases. For example, at $7.3 \mathrm{~kW}$ power, the flow has a zero nitrogen atom mole fraction, i.e., it is fully molecular. As the flow stream progresses toward the test sample, for a high temperature of $7000 \mathrm{~K}$, the level of dissociation increases, and hence the nitrogen atom number density, increases.

The total heat flux and temperature at the wall for these cases are shown in Figs. 18a and 18b, respectively. The mass removal flux is shown in Fig. 19. The wall heat flux, wall temperature, and the mass loss increase with the increase in the power. This increase is explained by the higher flux of nitrogen atoms at the test sample surface. Therefore, a higher number of atoms recombine at the surface catalytically and release the heat of recombination into the surface, thus increasing the heat flux and wall temperature along the test sample surface. Also, more carbon nitridation occurs (for the same nitridation efficiency), as a higher number of nitrogen atoms are 

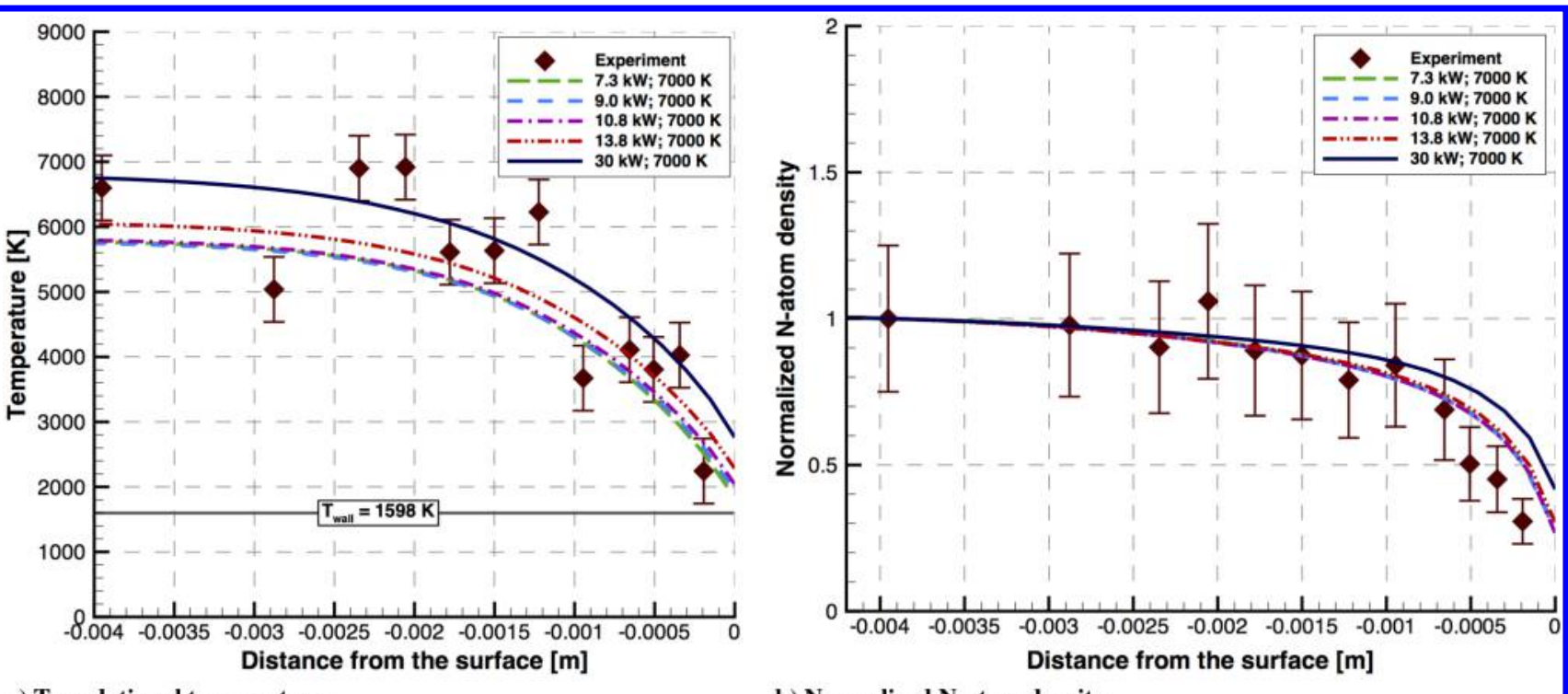

a) Translational temperature

b) Normalized $\mathrm{N}$-atom density

Fig. 15 Comparison of translational temperature and normalized $\mathrm{N}$-atom density along the stagnation line in the boundary layer for varying power.

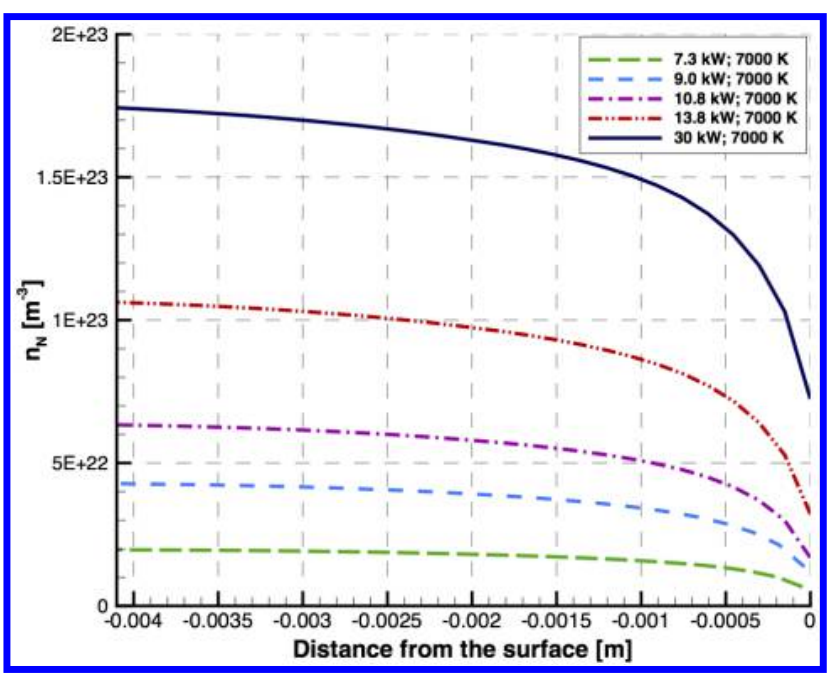

Fig. 16 Comparison of $\mathrm{N}$-atom number density along the stagnation line in the boundary layer for varying inlet power. available at the surface due to the higher flux of nitrogen atoms. Thus, higher mass loss is experienced at higher power.

The comparison between the stagnation point values and the mass loss rate for these cases, along with the experimentally measured values, is shown in Table 7 . There is an approximately $25 \%$ increase in heat flux both for an increase in power from 7.3 to $9.0 \mathrm{~kW}$ and from 9.0 to $10.8 \mathrm{~kW}$. The heat flux increases by approximately $56 \%$ when power is changed from 10.8 to $13.8 \mathrm{~kW}$, and an increase of about $110 \%$ is observed for a change from 13.8 to $30 \mathrm{~kW}$. There is a $6 \%$ increase in temperature at the wall for both an increase in power from 7.3 to $9.0 \mathrm{~kW}$ and from 9.0 to $10.8 \mathrm{~kW}$. The increase in temperature at the wall is approximately $12 \%$ for an increase in power from 10.8 to $13.8 \mathrm{~kW}$, and an increase of $21 \%$ is observed for a change from 13.8 to $30 \mathrm{~kW}$.

There is an approximately $145 \%$ increase in the mass loss rate when the power is increased from 7.3 to $9.0 \mathrm{~kW}$, a $56 \%$ increase for the variation in power from 9.0 to $10.8 \mathrm{~kW}$, a $105 \%$ increase for 10.8 to $13.8 \mathrm{~kW}$, and an increase of $155 \%$ is observed for a change from 13.8 to $30 \mathrm{~kW}$.

It can be concluded from these results that the translational temperature and the nitrogen atom density in the boundary layer, surface heat flux, wall temperature, and mass removal flux along the

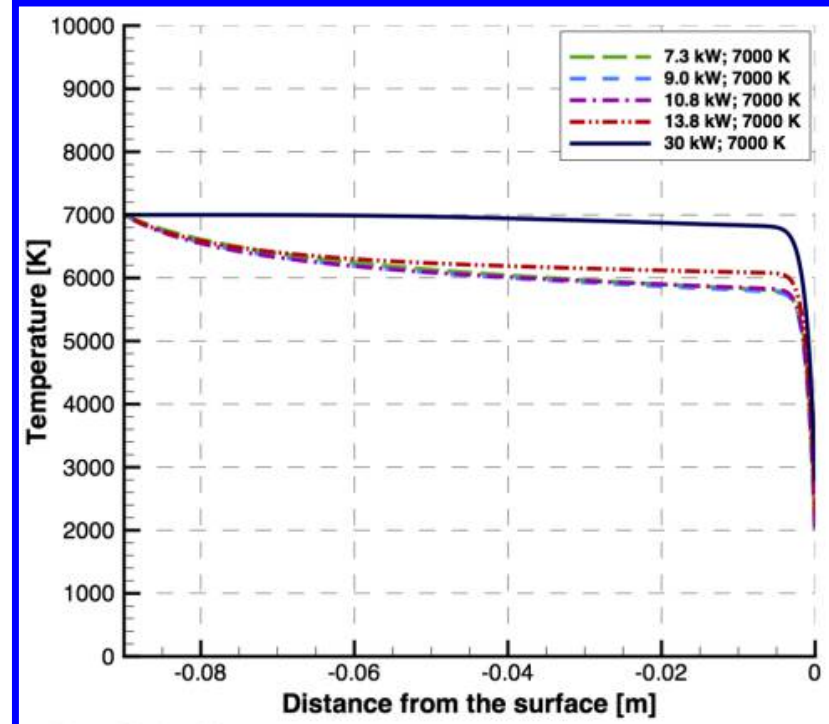

a) Translational temperature

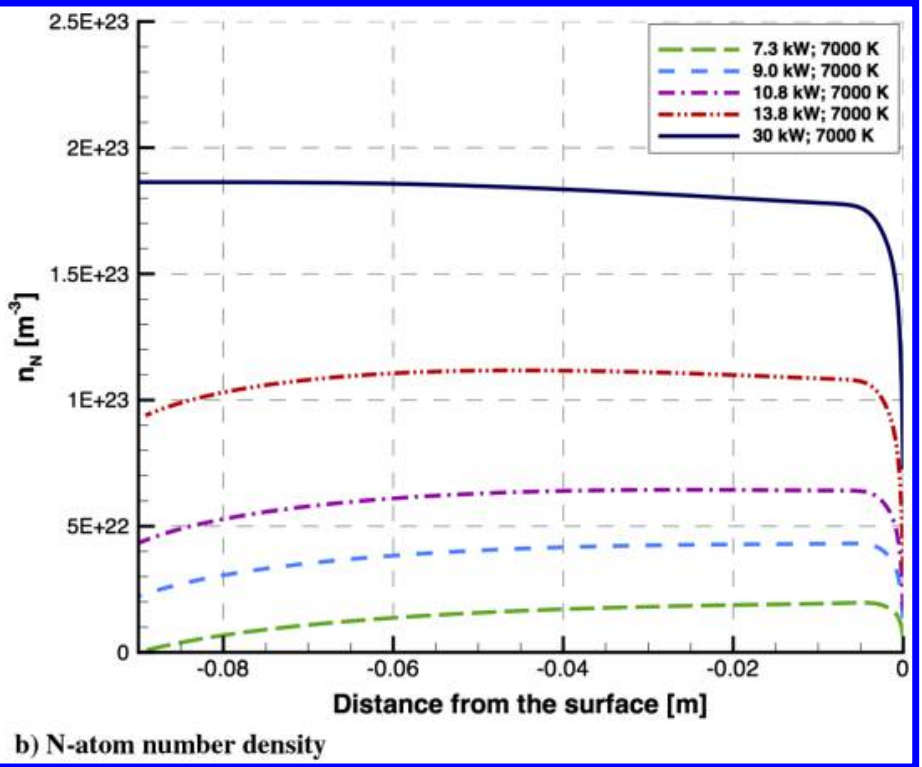

b) $\mathrm{N}$-atom number density

Fig. 17 Comparison of translational temperature and $\mathrm{N}$-atom number density along the stagnation line for varying power. 


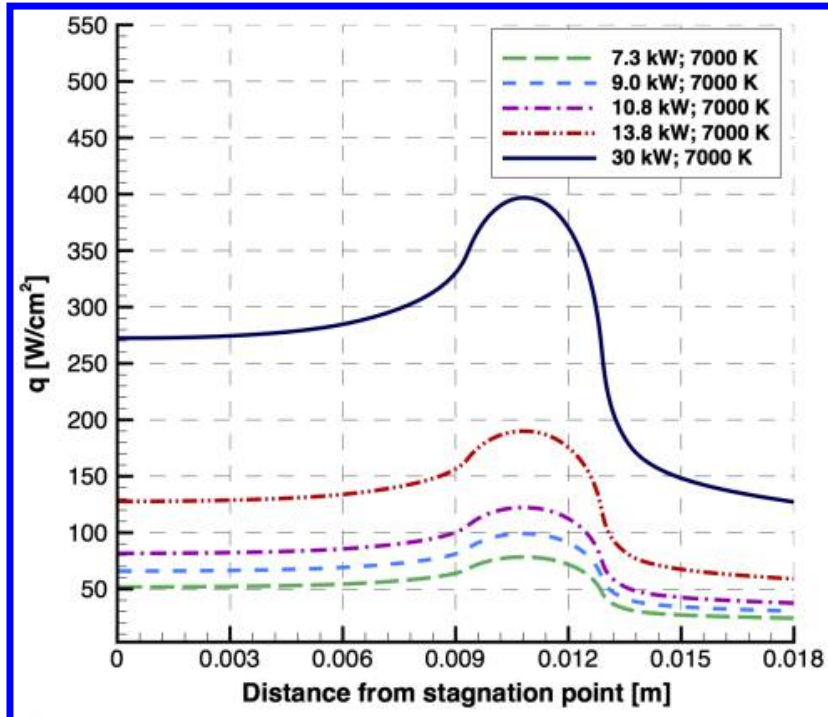

a) Total heat flux

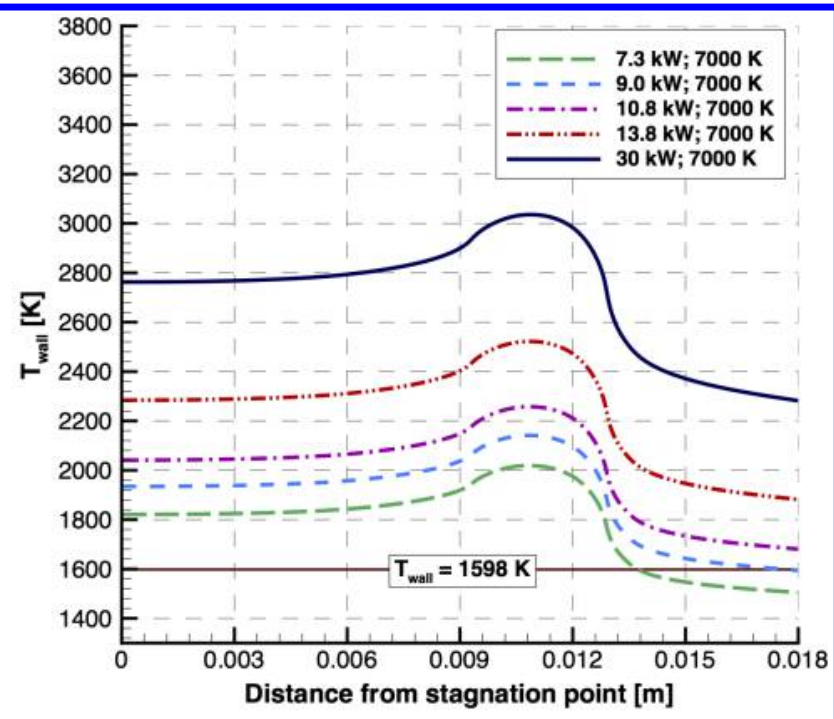

b) Surface temperature

Fig. 18 Comparison of wall heat flux and temperature between the computational results for varying power in the flow.

test sample surface are highly sensitive to power in the flow. Therefore, it is very important to experimentally characterize the power absorbed by the plasma in the ICP torch. The necessity of experimental measurements of absolute atom number densities is again shown by these results. The amount of nitrogen atom flux in the boundary layer directly affects the heat transferred, wall temperature, and mass loss rate. Therefore, the absolute number density is required to draw conclusions about the surface-chemistry models, as well as the surface reactions involved.

The computed stagnation point heat flux and mass loss for $9.0 \mathrm{~kW}$ power has good agreement with the experimental data. The stagnation point temperature for this case is $21 \%$ higher than the experimental value. The temperature at the wall is affected by the net heat transfer to the surface. The effects of conduction within the sample are therefore determined, and the results are presented in the following section.

\section{Accounting for Conduction into the Sample}

The net heat transfer to the wall is expected to have an impact on the surface properties. The contribution of conductive heat transfer within the sample is determined by using the material response code MOPAR. The coupling of MOPAR with LeMANS is described in Sec. III.C. The material properties of DFP2-grade Poco Graphite, Inc. graphite [15] are used in MOPAR. The properties specified are thermal conductivity, specific heat, and emissivity of graphite. The

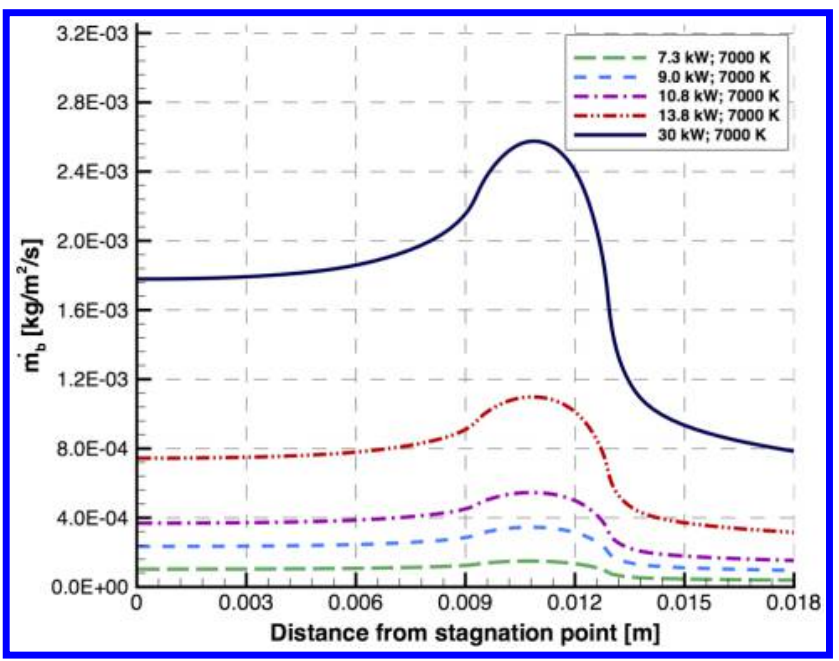

Fig. 19 Comparison of mass removal flux for varying power. case with $9.0 \mathrm{~kW}$ power has the best agreement with the experimental data for stagnation heat flux and mass loss. Therefore, this case is used to account for the effects of conduction within the sample.

An unstructured solid grid with 2100 cells is used. The boundary of the grid exposed to the flowfield is set as a wall boundary condition calculated by Eq. (17). The back wall temperature is set to $350 \mathrm{~K}$ based on the measured back temperature of the copper slug. $\mathbb{1}$ This value is used as the back temperature of the graphite test sample, as it is not measured experimentally. MOPAR runs for $300 \mathrm{~s}$ and then passes the respective wall temperature value to LeMANS. This time is chosen as the heat flux is measured experimentally after a $300 \mathrm{~s}$ exposure time to nitrogen plasma. The equations in LeMANS are solved for 200 iterations. The process is repeated until a converged steady-state solution is obtained.

The results from this simulation are compared with the results for the radiative equilibrium wall boundary condition. The contours for temperature in the flowfield and within the solid test sample are shown in Fig. 20. In this picture, the legend $T_{\text {flowfield }}$ shows the variation of translational temperature in the flowfield and $T_{\text {solid }}$ shows the variation of temperature within the solid. The flow enters from the ICP torch exit on the left and impinges on the graphite sample at the right. The front of the graphite sample is exposed to hot nitrogen plasma. The flowfield contours of temperature show the way in which the hot nitrogen plasma evolves on exiting the ICP torch. The temperature contours within the graphite sample show the temperature gradient within the sample. The sample is at the highest temperature in the front region that is exposed to hot nitrogen plasma, and it is the lowest at the back that is maintained at a $350 \mathrm{~K}$ backtemperature.

The stagnation line profiles for translational temperature and normalized nitrogen atom number density in the boundary layer are shown in Figs. $21 \mathrm{a}$ and $21 \mathrm{~b}$, respectively. It can be seen that there is no significant change in the temperature and normalized nitrogen atom number density in the boundary layer when conduction within the sample is included in the calculations. The reason for this is that the same flowfield conditions are employed in both the simulations. However, in the vicinity of the surface, the temperature drops and nitrogen atom density rises for the case with conduction, as compared to the radiative equilibrium case. The reason for this is explained by the surface properties.

The comparison for the total heat flux and temperature at the wall are shown in Figs. 22a and 22b, respectively. There is an approximately $5 \%$ increase in heat flux and $17 \%$ reduction in temperature when heat is allowed to conduct into the material. The temperature decrease results in an increase in conductive heat flux as the change in temperature at the wall increases. This drop in temperature at the wall reduces the temperature in the flow in the vicinity of the surface. 


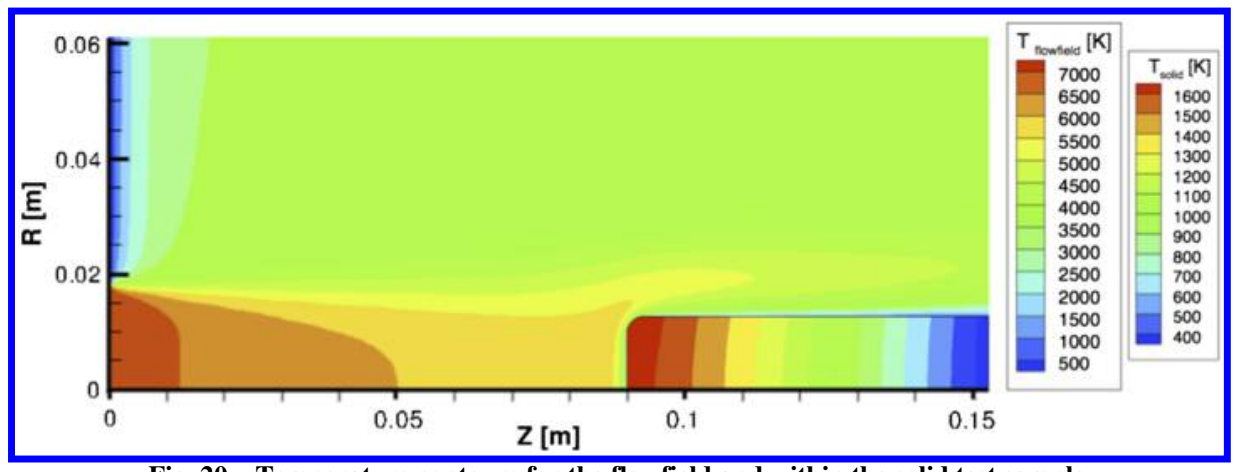

Fig. 20 Temperature contours for the flowfield and within the solid test sample.

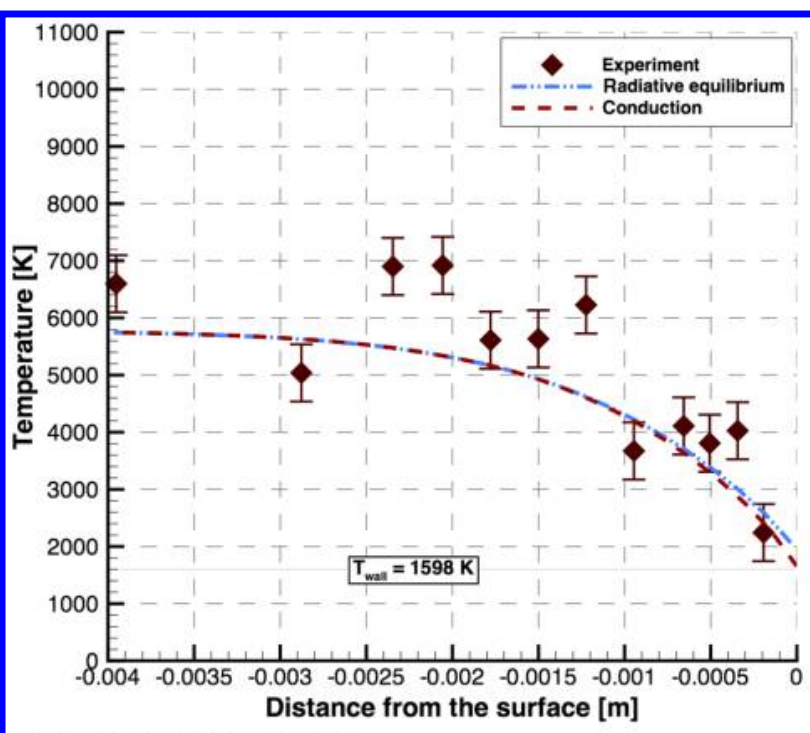

a) Translational temperature

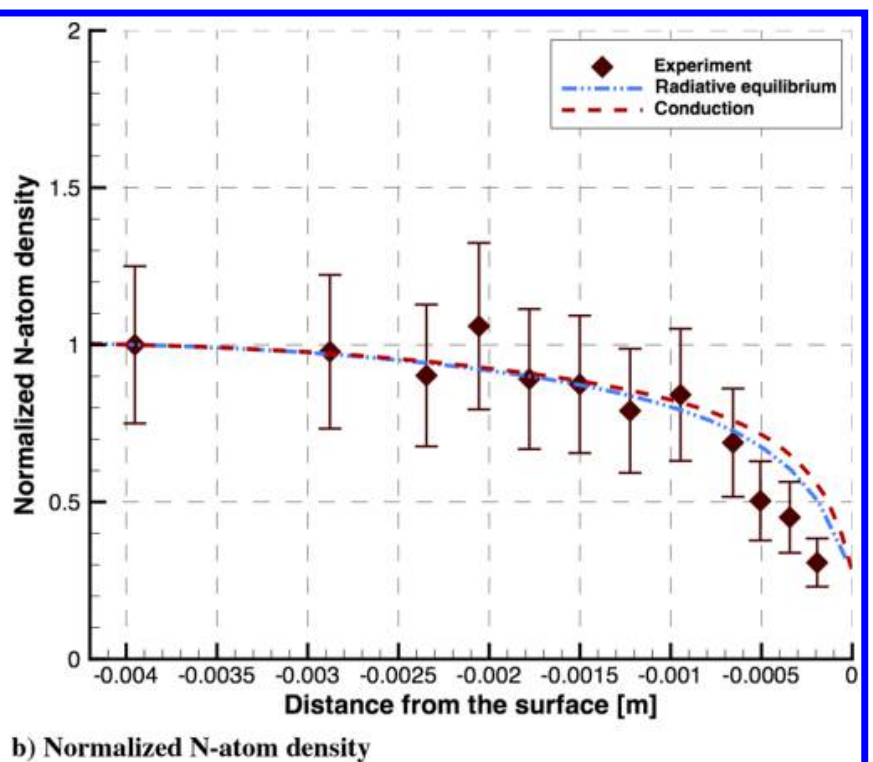

b) Normalized $\mathrm{N}$-atom density

Fig. 21 Comparison of temperature and normalized N-atom density for different energy balance conditions.

When the temperature decreases, the density of nitrogen atoms increases to maintain constant pressure in the flowfield.

The mass removal flux is shown in Fig. 23. There is no significant effect of conduction within the sample on the mass removal flux. The reason for this is that the mass removal flux depends on the nitrogen atom density and the square root of the temperature [Eq. (15)]. When conduction is taken into account, the wall temperature decreases but the nitrogen atom density increases. For the radiative equilibrium
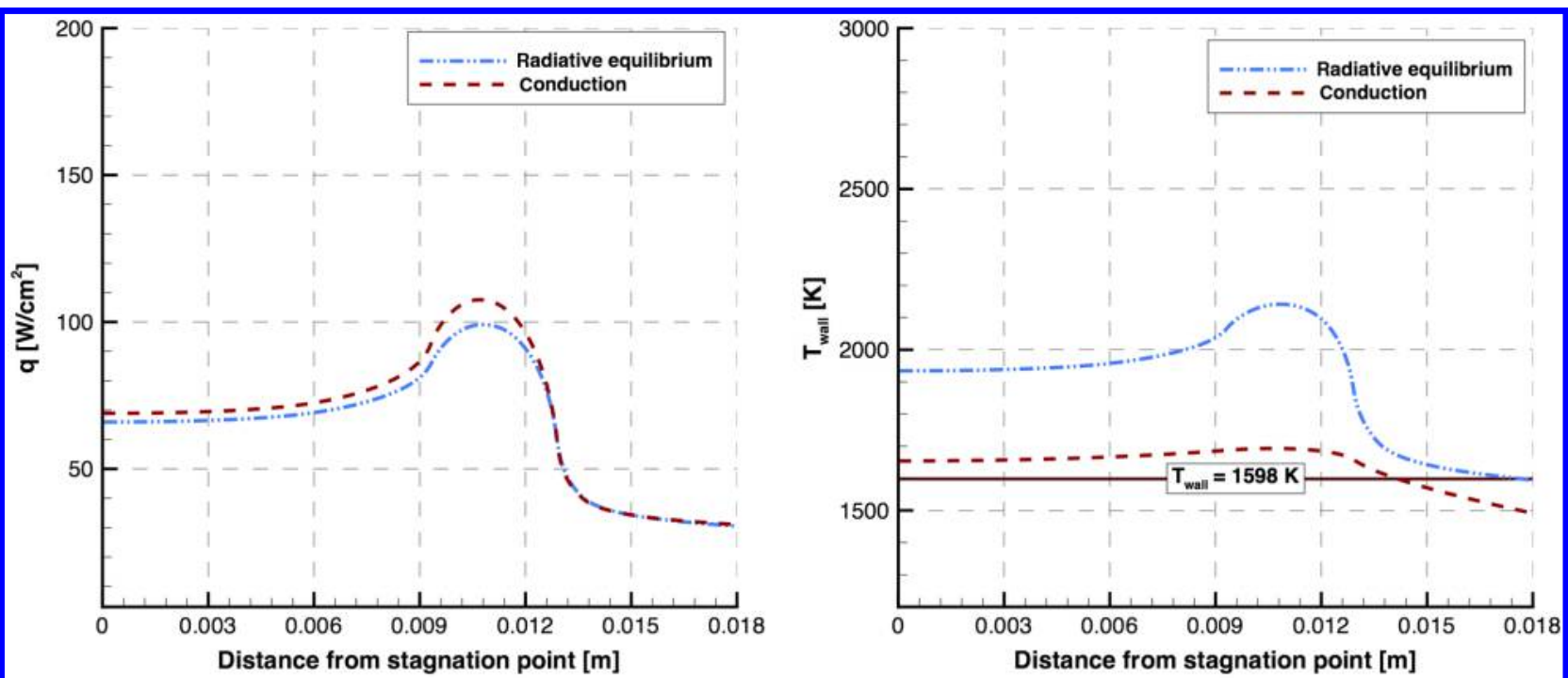

a) Total heat flux

b) Surface temperature

Fig. 22 Comparison of wall heat flux and temperature between the computational results for different energy balance conditions at the wall. 


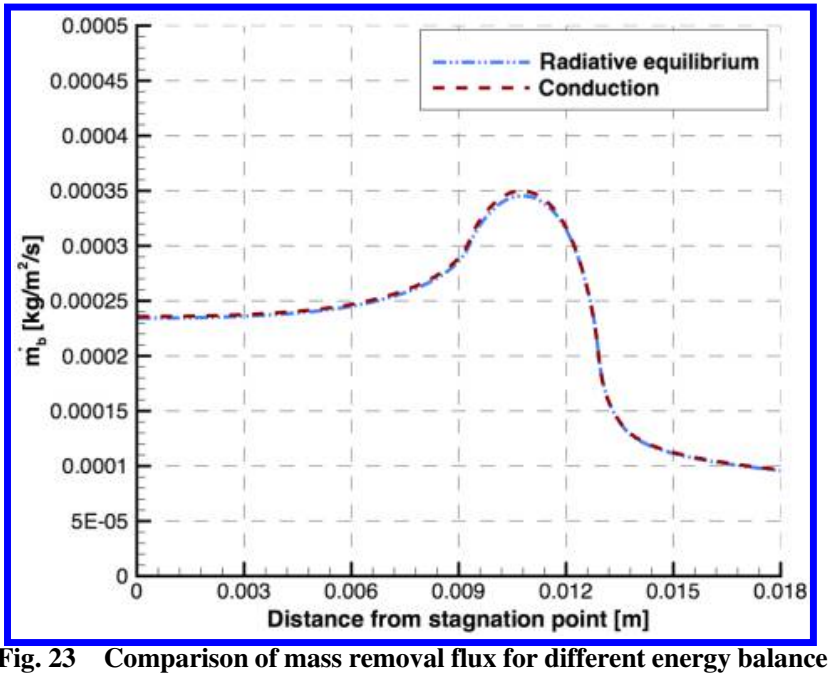
conditions at the wall.

Table 8 Stagnation point values and mass loss rates for different energy balance conditions at the wall

\begin{tabular}{lccccc}
\hline \hline & & \multicolumn{4}{c}{$q_{\text {stag, }}}$, \\
Power, kW & $T_{\text {inf }}, \mathrm{K}$ & $\mathrm{W} / \mathrm{cm}^{2}$ & $T_{\text {stag }}, \mathrm{K}$ & $\begin{array}{c}\text { Mass loss } \\
\text { rate, mg/s }\end{array}$ \\
\hline Radiative & 9.0 & 7000 & 66 & 1934 & 0.27 \\
equilibrium & & & & & \\
Conduction & 9.0 & 7000 & 69 & 1654 & 0.27 \\
Experiment & 13.8 & 7000 & $40-80$ & 1600 & $0.2-0.6$ \\
\hline \hline
\end{tabular}

case, the wall temperature increases but the nitrogen atom density decreases. Therefore, no change is observed in the mass removal flux between the two cases.

The comparison between the stagnation point values for these cases along with the experimental data are shown in Table 8 . There is an approximately $5 \%$ increase in stagnation point heat flux when conduction is included. There is an approximately $300 \mathrm{~K}$ drop (i.e., $17 \%$ ) in stagnation point temperature when conduction into the wall is accounted for. The mass removal rate remains unchanged. The stagnation point heat flux, temperature, and mass loss for $9.0 \mathrm{~kW}$ power with conductive heat transfer within the sample shows the best agreement with the experimental data. The results show that good agreement of computations with all experimental measurements is obtained if all the flow, surface, and material physics are included in the simulations using the input test conditions obtained as a result of the sensitivity study. It can also be concluded from these results that the power in the flow is much less than the estimated power of $13.8 \mathrm{~kW}$. As mentioned earlier, the back face temperature of the graphite test sample is not measured experimentally. Therefore, sensitivity of the surface properties to the backtemperature is determined. A simulation is performed for a back face temperature of $400 \mathrm{~K}$ for the same flowfield conditions of $9.0 \mathrm{~kW}$. The stagnation point temperature obtained from the simulations for this case is $1670 \mathrm{~K}$, which represents a $1 \%$ increase for a $50 \mathrm{~K}$ increase in the backtemperature. No change is observed in the stagnation point heat flux.

\section{Conclusions}

The major objective of this work was to investigate surfacechemistry processes using a coupled computational fluid dynamicssurface-chemistry model and assess the accuracy of the model using experimental data. Significant discrepancies were observed between the computational and experimental results. Therefore, another objective was to understand the sensitivities of flow and surface parameters to variations in testing input conditions. A sensitivity study was performed to understand the effects of various inputs as well as physical modeling parameters on the subsonic high-enthalpy nitrogen flow in the boundary layer in front of a graphite sample and its surface properties. The numerical simulations in this work were conducted using the Navier-Stokes computational fluid dynamics code LeMANS developed at the University of Michigan. The flow physics is modeled for thermochemical nonequilibrium flow. The surface-chemistry processes considered were the nitrogen atom recombination on the wall due to surface catalysis along with the carbon nitridation reaction where the carbon from the surface reacts with the impinging nitrogen atoms. Experimental data for flow and surface properties from tests conducted in the $30 \mathrm{~kW}$ inductively coupled plasma torch facility at the University of Vermont were used for the evaluations of the computations for surface-chemistry processes.

It was observed that, using the expected power and efficiency of the inductively coupled plasma torch and assuming chemical equilibrium at torch exit, there is too much energy in the flow, and the predicted heating, surface temperature, recession rates, and the flowfield properties are much higher than the actually measured experimental data. It was shown that a good agreement of computations with all experimental measurements was obtained only if all the flow, surface, and material physics were included in the simulations using the input test conditions obtained as a result of the sensitivity study. It was identified that true validation of the surfacechemistry models requires absolute number density measurements. The relative number density measurements are not sufficient for validation of these models. It was determined that validation of such simulations also requires better characterization of the power absorbed by the plasma in the ICP torch, as the effect of varying flow power for a constant inlet temperature on flow properties and surface properties is highly significant. The power was shown to be in the range of $9 \mathrm{~kW}$ to get reasonable agreement with the measured data, which indicates that the losses in the ICP torch need to be quantified. It was shown that the effect of varying the inlet temperature for constant power on flow properties and surface properties is negligible, which shows that the surface and flowfield properties are mainly dependent on the power of the flow. It is shown that there is a significant drop in surface temperature when the contribution of material response is included in the simulations.

Several other measurements needed from experiments to allow better validation of modeling were identified. To better model the material response, the backtemperature value is critical. An analysis to determine the sensitivity of the surface properties to the backtemperature was also performed in this study. It was concluded from this analysis that the surface temperature increased for a higher back face temperature. The test sample is water cooled and will affect the temperature measurements on the surface. Therefore, a backtemperature measurement will allow for a better estimate of surface properties. Also, measurement of the flow temperature and absolute species densities at the inlet of the test chamber will allow for better estimation of inlet conditions. It was seen in all the computational results that the effect of inlet flow composition is very significant on both the flow and surface properties.

\section{Acknowledgments}

The authors gratefully acknowledge funding for this work through Air Force Office of Scientific Research grant FA-9550-11-1-0309. The authors also acknowledge critical information provided for this research by Doug Fletcher and his graduate students Andrew Lutz and Walt Owens at the University of Vermont.

\section{References}

[1] Anderson, J. D., Hypersonic and High-Temperature Gas Dynamics, 2nd ed., AIAA, Reston, VA, Nov. 2006, pp. 16-17.

[2] Marschall, J., and Fletcher, D. G., "High-Enthalpy Test Environments, Flow Modeling and In Situ Diagnostics for Characterizing Ultra-High Temperature Ceramics," Journal of the European Ceramic Society, Vol. 30, No. 11, 2010, pp. 2323-2336. doi:10.1016/j.jeurceramsoc.2010.01.010

[3] Laub, B., and Venkatapathy, E., "Thermal Protection System Technology and Facility Needs for Demanding Future Planetary Missions," Proceedings of the International Workshop Planetary Probe Atmospheric Entry and Descent Trajectory Analysis and Science, 
edited by Wilson, A., ESA, SP-544, ESA Publ. Division, Noordwijk, The Netherlands, Oct. 2003, pp. 239-247.

[4] Harvey, G. A., "Simulated Reentry Heating by Torching," NASA CP2008-214164, NASA Langley Research Center, Hampton, VA, Oct. 2008.

[5] Robinson, J., and Wurster, K., "Trajectory and Aeroheating Environment Development and Sensitivity Analysis for Capsule-Shaped Vehicles," AIAA Paper 2006-7949, 2006.

-[6] Trumble, K. A., Cozmuta, I., Sepka, S., Jenniskens, P., and Winter, M., "Postflight Aerothermal Analysis of Stardust Sample Return Capsule," Journal of Spacecraft and Rockets, Vol. 47, No. 5, Sept.-Oct. 2010, pp. $765-774$ doi: $10.2514 / 1.41514$

- [7] Milos, F. S., and Rasky, D. J., "Review of Numerical Procedures for Computational Surface Thermochemistry," Journal of Thermophysics and Heat Transfer, Vol. 8, No. 1, Jan.-March 1994, pp. 24-34. doi: $10.2514 / 3.497$

[8] Bianchi, D., "Modeling of Ablation Phenomena in Space Applications," Ph.D. Thesis, Univ. of Rome "La Sapienza", Rome, 2008.

-[9] Lutz, A., Owens, W., Meyers, J., Fletcher, D., and Marschall, J., "Investigation of $\mathrm{CN}$ Production from Carbon Materials in Nitrogen Plasmas," AIAA Paper 2011-0901, Jan. 2011.

[10] Owens, W. P., Uhl, J., Dougherty, M., Lutz, A., Meyers, J., and Fletcher, D. G., "Development of a $30 \mathrm{~kW}$ Inductively Coupled Plasma Torch for Aerospace Material Testing," AIAA Paper 2010-4322, June 2010.

[11] Chazot, O., Panerai, F., Muylaert, J., and Thoemel, J., "Catalysis Phenomena Determination in Plasmatron Facility for Flight Experiment Design," AIAA Paper 2010-1248, Jan. 2010.

[12] Barbante, P., "Heat Flux Duplication Between Ground Facility and Hypersonic Flight," Journal of Thermophysics and Heat Transfer, Vol. 23, No. 4, Oct.-Dec. 2009, pp. 684-692. doi: $10.2514 / 1.35808$

[13] Barbante, P., and Chazot, O., "Flight Extrapolation of Plasma Wind Tunnel Stagnation Region Flowfield," Journal of Thermophysics and Heat Transfer, Vol. 20, No. 3, July-Sept. 2006, pp. 493-499. doi: $10.2514 / 1.17185$

[14] Kolesnikov, A. F., "Extrapolation from High Enthalpy Tests to Flight Based on the Concept of Local Heat Transfer Simulation," NATO RTOEN-8, Neuilly-sur-Seine Cedex, France, 1999, pp. 8B/1-14.

[15] Sheppard, R. G., Mathes, D. M., and Bray, D. J., Properties and Characteristics of Graphite, Poco Graphite, Decatur, TX, Nov. 2001, pp. 11.1-14.2.

[16] Owens, W. P., Meyers, J. M., Fletcher, D. G., Corso, J. A. D., and Calomino, A. M., "Flexible TPS Surface Catalysis Testing in a $30 \mathrm{~kW}$ ICP Torch Facility," 43rd AIAA Thermophysics Conference, AIAA Paper 2012-3095, June 2012.

[17] Lutz, A. J., "Investigation of CN Production from Carbon Materials in Nitrogen Plasmas," M.S. Thesis, Univ. of Vermont, Burlington, VT, Oct. 2011

[18] Anna, A., "Numerical Modeling of Surface Chemistry Processes for Hypersonic Entry Environments," Ph.D. Thesis, Univ. of Michigan, Ann Arbor, MI, 2013.

[19] Martin, A., Scalabrin, L. C., and Boyd, I. D., "High Performance Modeling of Atmospheric Re-Entry Vehicles," Journal of Physics: Conference Series, Vol. 341, No. 1, 2012, Paper 012002. doi:10.1088/1742-6596/341/1/012002

[20] Scalabrin, L. C., and Boyd, I. D., "Numerical Simulation of Weakly Ionized Hypersonic Flow for Reentry Configurations," AIAA Paper 2006-3773, June 2006.

-[21] Martin, A., and Boyd, I. D., "CFD Implementation of a Novel CarbonPhenolic-in-Air Chemistry Model for Atmospheric Re-Entry," AIAA Paper 2011-143, Jan. 2011.

[22] Park, C., "Assessment of Two-Temperature Kinetic Model for Ionizing Air," AIAA Paper 1987-1574, June 1987.

[23] MacCormack, R., and Candler, G., "The Solution of the Navier-Stokes Equations Using Gauss-Siedel Line Relaxation," Computers and Fluids, Vol. 17, No. 1, 1989, pp. 135-150. doi:10.1016/0045-7930(89)90012-1

[24] Karypis, G., and Kumar, V., METIS: A Software Package for Partitioning Unstructured Graphs, Partitioning Meshes, and Computing Fill-Reducing Orderings of Sparse Matrices, Univ. of Minnesota, Minneapolis, MN, 1998.

[25] Baldwin, B. S., and Lomax, H., "Thin Layer Approximation and Algebraic Model for Separated Turbulent Flows," AIAA Paper 19780257, Jan. 1978.
[26] Wilke, C. R., "A Viscosity Equation for Gas Mixtures," Journal of Chemical Physics, Vol. 18, No. 4, 1950, pp. 517-519. doi:10.1063/1.1747673

[27] Blottner, F. G., Johnson, M., and Ellis, M., "Chemically Reacting Viscous Flow Program for Multi-Component Gas Mixtures," Sandia Labs. Rept. Sc-rr-70-754, Albuquerque, NM, 1971.

[28] Vincenti, W. G., and Kruger, C. H., Introduction to Physical Gas Dynamics, Krieger, Malabar, FL, 2002, p. 21.

[29] Gupta, R., Yos, J., Thompson, R., and Lee, K., "A Review of Reaction Rates and Thermodynamic and Transport Properties for an 11-Species Air Model for Chemical and Thermal Nonequilibrium Calculations to 30,000 K," NASA RP-1232, 1990.

[30] Scalabrin, L. C., "Numerical Simulation of Weakly Ionized Hypersonic Flow over Reentry Capsules," Ph.D. Thesis, Univ. of Michigan, Ann Arbor, MI, 2007.

[31] Holman, T. D., and Boyd, I. D., "Numerical Investigation of the Effects of Continuum Breakdown on Hypersonic Vehicle Surface Properties," AIAA Paper 2008-3928, June 2008.

[32] Deschenes, T. R., "Extension of a Modular Particle-Continuum Method for Nonequilibrium, Hypersonic Flows," Ph.D. Thesis, Univ. of Michigan, Ann Arbor, MI, 2011.

[33] Alkandry, H., "Aerodynamic Interactions of Propulsive Deceleration and Reaction Control System Jets on Mars-Entry Aeroshells," Ph.D. Thesis, Univ. of Michigan, Ann Arbor, MI, 2012.

[34] Rudy, D. H., and Strikwerda, J. C., "Boundary Conditions for Subsonic Compressible Navier-Stokes Calculations," Computers and Fluids, Vol. 9, No. 3, 1981, pp. 327-338. doi:10.1016/0045-7930(81)90005-0

[35] Scott, C. D., "Wall Catalytic Recombination and Boundary Conditions in Nonequilibrium Hypersonic Flow-With Applications," The Third Joint Europe/US Short Course in Hypersonic Flow, Birkhäuser, Boston, Oct. 1990, pp. 176-250. doi:10.1007/978-1-4612-0371-1 6

[36] Marschall, J., and MacLean, M., "Finite-Rate Surface Chemistry Model, I: Formulation and Reaction System Examples," AIAA Paper 2011-3783, June 2011.

[37] MacLean, M., Marschall, J., and Driver, D. M., "Finite-Rate Surface Chemistry Model, II: Coupling to Viscous Navier-Stokes Code," AIAA Paper 2011-3784, June 2011.

[38] Alkandry, H., Farbar, E. D., and Boyd, I. D., "Evaluation of Finite-Rate Surface Chemistry Models for Simulation of the Stardust Reentry Capsule," AIAA Paper 2012-2874, June 2012.

[39] Sutton, K., and Gnoffo, P. A., "Multi Component Diffusion with Application to Computational Aerothermodynamics," AIAA Paper 19982575, 1998.

[40] Martin, A., and Boyd, I. D., "Non-Darcian Behavior of Pyrolysis Gas in a Thermal Protection System," Journal of Thermophysics and Heat Transfer, Vol. 24, No. 1, Jan.-March 2010, pp. 60-68. doi: $10.2514 / 1.44103$

[41] Wiebenga, J. E., and Boyd, I. D., "Computation of Multi-Dimensional Material Response Coupled to Hypersonic Flow," 43rd AIAA Thermophysics Conference, AIAA Paper 2012-2873, June 2012.

[42] Pointwise, Software Package, Ver. 16.04, Pointwise, Ft. Worth, TX, 2011.

[43] Lutz, A., Meyers, J., Owens, W., Smith, S., and Fletcher, D. G., "Experimental Analysis of Carbon Nitridation and Oxidation Efficiency with Laser-Induced Fluorescence," 51st AIAA Aerospace Sciences Meeting, AIAA Paper 2013-0924, Jan. 2013.

[44] Gordeev, A. N., Kolesnikov, A. F., and Yakushin, M. I., "Effect of Surface Catalytic Activity on Nonequilibrium Heat Transfer in a Subsonic Jet of Dissociated Nitrogen," Fluid Dynamics, Vol. 20, No. 3 , 1985, pp. 478-484. doi:10.1007/BF01050006

[45] Driver, D. M., and MacLean, M., "Improved Predictions of PICA Recession in Arc Jet Shear Tests," AIAA Paper 2011-0141, Jan. 2011.

[46] Anna, A., Boyd, I. D., Colombo, V., Ghedini, E., Sanibondi, P., Boselli, M., and Gherardi, M., "Computational Modeling of Surface Catalysis for Graphite Exposed to High-Enthalpy Nitrogen Flow," RTO-AVT-199, Jan. 2012.

[47] Gordon, S., and McBride, B. J., Computer Program for Calculation of Complex Chemical Equilibrium Compositions and Applications, NASA Reference Publ. 1311, 1996.

[48] Lutz, A., Owens, W., Meyers, J., Fletcher, D., and Marschall, J., "Investigation of $\mathrm{CN}$ Production from Carbon Materials in Nitrogen Plasmas," AIAA Paper 2011-0901, Jan. 2011. 
This article has been cited by:

1. Nicholas Martin, Jason M. Meyers, Douglas G. Fletcher, David Dang, Iain D. Boyd. Investigation of Pyrolyzing Ablators Using a Gas Injection Probe . [Citation] [PDF] [PDF Plus] 